\title{
Asymptotics of Arbitrary Order for a Thin Elastic Clamped Plate, I. Optimal Error Estimates
}

\author{
Monique Dauge \& Isabelle Gruais
}

\begin{abstract}
This paper is the first of a series of two, where we study the asymptotics of the displacement in a thin clamped plate made of a rigid "monoclinic" material, as the thickness of the plate tends to 0 . The combination of a polynomial Ansatz (outer expansion) and of a boundary layer Ansatz (inner expansion) yields a complete multi-scale asymptotics of the displacement and leads to optimal error estimates in energy norm. We investigate the polynomial Ansatz in Part I, and the boundary layer Ansatz in Part II.

If $\varepsilon$ denotes the small parameter in the geometry, we first construct the algorithm for an infinite "even" Ansatz involving only even powers of $\varepsilon$, which is a natural extension of the usual Kirchhoff-Love Ansatz. The boundary conditions of the clamped plate being only satisfied at the order 0 , we try to compensate for them by boundary layer terms: we rely on a result proved in Part II giving necessary and sufficient conditions for the exponential decay of such terms. In order to fulfill these conditions, the constructive algorithm for the boundary layer terms has to be combined with an "odd" polynomial Ansatz. The outcome is a two-scale asymptotics involving all nonnegative powers of $\varepsilon$, the in-plane space variables $x_{\alpha}$, the transverse scaled variable $x_{3}$ and the quickly varying variable $r / \varepsilon$ where $r$ is the distance to the clamped part of the boundary.
\end{abstract}

To appear in Asymptotic Analysis 


\section{CONTENTS}

1 Setting the problem. Statements of the main results. 4

1.1 The Plate Problem . . . . . . . . . . . . . . . . . 4

1.2 The scaling ........................... 5

1.3 Related questions . . . . . . . . . . . . . . . . 6 6

1.4 Polynomial Ansätze and boundary layers . . . . . . . . . . . . . 6

1.5 Outline of the paper and main results . . . . . . . . . . . . 8

2 The "even" Ansatz 12

2.1 The sequence of boundary value problems . . . . . . . . . . . . . 12

2.2 Kirchhoff-Love displacements . . . . . . . . . . . . . . . . . . . 13

2.3 Existence and uniqueness of an even Ansatz . . . . . . . . . . . 16

2.4 An even Ansatz for problem (1.6) . . . . . . . . . . . . . . . 19

3 The reduced-normal problem on a semi-infinite strip for the determination of the boundary layer Ansatz 20

3.1 Guess of the boundary layers . . . . . . . . . . . . . . . . . . . . 20

3.2 Position of the reduced-normal problem . . . . . . . . . . . . . 21

3.3 Behavior at infinity of solutions of the reduced-normal problem . . . . 23

4 Complete asymptotics $\quad 24$

4.1 The first terms in the boundary layer series . . . . . . . . . . . . . . . 24

4.2 Asymptotic expansion of arbitrary order . . . . . . . . . . . . 25

4.3 Regularity of the terms of the polynomial Ansatz . . . . . . . . . 28

5 Error estimates in energy norm $\quad 29$

5.1 The main error estimate. . . . . . . . . . . . . . . . . . . 29

5.2 Proof of Theorem $(5.1) \ldots \ldots \ldots \ldots$

$\begin{array}{ll}\text { References } & 32\end{array}$ 


\section{INTRODUCTION}

The problem of the construction of two-dimensional models for thin plates was treated for a long time: the famous Kirchhoff model was stated around 1850, and the Reissner-Mindlin model, about 100 years later. The question of the validity of such models consists in the evaluation of the distance between the solution of the "true" three-dimensional problem and the solution deduced from the two-dimensional model: the faster the decay of this distance is as the plate thickness tends to 0 , the better is the two-dimensional model.

The answer to such a question requires an asymptotic analysis. This is quite difficult, due to the fact that the small parameter $\varepsilon$ intervenes as a parameter of singular perturbation: as $\varepsilon$ tends to 0 , the three-dimensional problem looses a part of its ellipticity (with respect to the in-plane space variables). As a consequence, a simple polynomial Ansatz with respect to $\varepsilon$ cannot fulfill all boundary conditions, especially the Dirichlet condition of the hard clamped plate. Thus, we can expect the combination of an outer expansion (polynomial Ansatz) and of an inner expansion (boundary layer Ansatz).

The first approach is the construction of infinite formal asymptotic expansions (i.e. without error estimates) for both the inner and outer parts. We can quote the papers [18] by K. O. Friedrichs \& R. F. Dressler in 1961 and [19] by A. L. Gol'Denveizer in 1962, and more recently [20] by R. D. Gregory \& F. Y. WAn.

The other approach is to obtain rigorous bounds of the error, at least for the first terms. Such an approach is initiated by P. G. Ciarlet and P. Destuynder in [8], [14]. We can also quote the more recent work [32] by S. A. NAZARov \& I. S. Zorin. See also the review by P. G. Ciarlet [7, Ch.3].

Here, we provide a sort of synthesis of the two approaches: we construct the terms of arbitrary order in the inner and outer expansions, and we prove optimal estimates between the three-dimensional solution and any truncated series of the asymptotics: these estimates are optimal in the sense that the order of the error is exactly the norm of the first neglected terms. In this first part we insist on the construction algorithm of the expansions and the estimates in lower order norms $\left(H^{1}, L^{2}\right.$ for the displacement field, $L^{2}$ for the strain tensor). In the second part, we prove the results about the exponential decay of the boundary layer terms that we need here, and by a complementary study of the behavior at the edges of the plate, we obtain estimates in higher order norms, and we deduce estimates in $L^{\infty}$ norm and in special tensorized norms for the stress tensor.

\section{ACKNOWLEDGEMENTS}

The authors are glad to thank P. G. Ciarlet and M. Costabel for valuable discussions and remarks.

This work is part of the Human Capital and Mobility Program "Shells: Mathematical Modeling and Analysis, Scientific Computing" of the Commission of the European Communities (Contract n ${ }^{\circ}$ ERBCHRXCT940536). 


\section{SETTING THE PROBLEM. STATEMENTS OF THE MAIN RESULTS.}

\subsection{The Plate Problem}

We study the behavior of a thin elastic plate made of a homogeneous material which will be characterized through its rigidity matrix $A$. Practically, we consider a plate represented by an open set

$$
\Omega^{\varepsilon}=\omega \times(-\varepsilon, \varepsilon) \subset \mathbb{R}^{3},
$$

where $\omega \subset \mathbb{R}^{2}$ is a bounded plane domain with a smooth boundary ( $\omega$ is the middle surface of the plate), and where the parameter $\varepsilon>0$ stands for half the thickness. We consider the case when the plate $\Omega^{\varepsilon}$ is clamped along its lateral face

$$
\Gamma_{0}^{\varepsilon}=\partial \omega \times(-\varepsilon, \varepsilon) .
$$

The unknown of the problem is the displacement $u^{\varepsilon}$ which belongs to a space $V\left(\Omega^{\varepsilon}\right)$ of so-called "admissible displacements" having a finite elastic energy, varying in $\bar{\Omega}^{\varepsilon}$ and taking their values in $\mathbb{R}^{3}$; since we assume that the plate is clamped along its lateral face, admissible displacements are zero on $\Gamma_{0}^{\varepsilon}$. Hence:

$$
V\left(\Omega^{\varepsilon}\right)=\left\{v=\left(v_{1}, v_{2}, v_{3}\right) \in H^{1}\left(\Omega^{\varepsilon}\right)^{3} \mid \quad v=0 \quad \text { on } \quad \Gamma_{0}^{\varepsilon}\right\} .
$$

For an admissible displacement $u^{\varepsilon}$, the stress tensor is defined by the formula

$$
\sigma^{\varepsilon}=\left(\sigma_{i j}^{\varepsilon}\right):=A e\left(u^{\varepsilon}\right),
$$

where $A$ is the rigidity matrix and $e\left(u^{\varepsilon}\right)=\left(e_{i j}\left(u^{\varepsilon}\right)\right)_{i j}$ is the linearized strain tensor defined by $e_{i j}(v)=\frac{1}{2}\left(\partial_{i} v_{j}+\partial_{j} v_{i}\right)$. We will take a rigidity matrix of a quite general form, but we assume that its coefficients do not depend on $\varepsilon$ (if not, we could obtain different asymptotic models).

We make the assumption [6] that we can take the linearized equations of elasticity. The displacement $u^{\varepsilon}$ of the clamped plate corresponding to volume forces $f^{\varepsilon}$ is the unique solution of the variational elasticity problem:

$$
\begin{aligned}
u^{\varepsilon} & \in V\left(\Omega^{\varepsilon}\right), \\
\forall v^{\varepsilon} & \in V\left(\Omega^{\varepsilon}\right), \quad \int_{\Omega^{\varepsilon}} A e\left(u^{\varepsilon}\right): e\left(v^{\varepsilon}\right)=\int_{\Omega^{\varepsilon}} f^{\varepsilon} \cdot v^{\varepsilon},
\end{aligned}
$$

where the dots : denote the usual scalar product for $3 \times 3$ matrices associated with the Frobenius norm $a: b=\sum_{i j} a_{i j} b_{i j}$. The displacement $u^{\varepsilon}$ actually satisfies Neumann condition (zero normal traction) on the two "horizontal" faces of the plate $\omega \times\{ \pm \varepsilon\}$. Our aim is to study the behavior of $u^{\varepsilon}$ as $\varepsilon \rightarrow 0$ when $f^{\varepsilon}$ depends on $\varepsilon$ in a "natural" way. Indeed, we could obtain similar results with prescribed normal tractions on the two "horizontal" faces of the plate. 
The $9 \times 9$ rigidity tensor $A$, with coefficients $A_{i j k l}$, satisfies the usual symmetry relations $A_{i j k l}=A_{j i k l}=A_{i j l k}=A_{k l i j}$ and is supposed to be uniformly positive definite, namely:

$$
\forall x^{\varepsilon} \in \bar{\Omega}^{\varepsilon}, \forall\left(t_{i j}\right) \in \mathbb{R}^{9} \text { s.t. } t_{i j}=t_{j i}, \quad A_{i j k l}\left(x^{\varepsilon}\right) t_{k l} t_{i j} \geq c t_{i j}^{2}
$$

where $c>0$ is a positive constant. Moreover, as in [16], [38] we assume that

$$
\begin{cases}A_{\alpha \beta \gamma 3}=0 & \forall \alpha, \beta, \gamma \in\{1,2\} \\ A_{\alpha 333}=0 & \forall \alpha \in\{1,2\} .\end{cases}
$$

The relations (1.3) are satisfied for any isotropic material, and also for an orthotropic material whose orthotropy axes are either normal or tangent to the plane containing $\omega$. Property (1.3) is referred in [38] as the characterization of monoclinic materials, and it is noted there that this is the weakest assumption so that the system of elasticity can be uncoupled into a bending and a membrane problems.

To simplify our forthcoming analysis, we moreover assume at this stage that the coefficients of $A$ do not depend on the "vertical" coordinate $x_{3}$. But, as in [39] for a linear heat conduction problem, the case of laminated materials might be worthwhile studying.

\section{$1.2 \quad$ The scaling}

Problem (1.1) was already studied by P. G. Ciarlet and P. Destuynder [8] [14] by means of an asymptotic expansion for the displacement-stress approach. See also [7] for a review on the subject. First, a scaling is worked out to get rid of the dependence of the reference set $\Omega^{\varepsilon}$ on the parameter $\varepsilon>0$. So, the problem is set in an equivalent form on the reference set $\Omega=\omega \times(-1,1)$, which is the image of $\Omega^{\varepsilon}$ through a dilation along the normal direction to the plane containing $\omega$ :

$$
x^{\varepsilon}=\left(x_{1}^{\varepsilon}, x_{2}^{\varepsilon}, x_{3}^{\varepsilon}\right) \in \Omega^{\varepsilon} \quad \longmapsto \quad x=\left(x_{1}^{\varepsilon}, x_{2}^{\varepsilon}, \varepsilon^{-1} x_{3}^{\varepsilon}\right) \in \Omega .
$$

It is convenient to preserve the structure of the former problem which was associated with the tensor $e\left(u^{\varepsilon}\right)$. So we introduce a "scaled" linearized strain tensor denoted by $\kappa(\varepsilon)(v)$ for any function $v \in H^{1}(\Omega)^{3}$ :

$$
\kappa_{\alpha \beta}(\varepsilon)(v)=e_{\alpha \beta}(v), \quad \kappa_{\alpha 3}(\varepsilon)(v)=\varepsilon^{-1} e_{\alpha 3}(v), \quad \kappa_{33}(\varepsilon)(v)=\varepsilon^{-2} e_{33}(v),
$$

where we use the convention that the Greek indices $\alpha$ and $\beta$ span the set $\{1,2\}$. Correspondingly, the convenient scaling of the components of $u^{\varepsilon}$ writes

$$
u_{\alpha}(\varepsilon)(x)=u_{\alpha}^{\varepsilon}\left(x^{\varepsilon}\right), \quad u_{3}(\varepsilon)(x)=\varepsilon u_{3}^{\varepsilon}\left(x^{\varepsilon}\right),
$$

for which the resulting scaled displacement $u(\varepsilon)$ satisfies

$$
e\left(u^{\varepsilon}\right)\left(x^{\varepsilon}\right)=\kappa(\varepsilon)(u(\varepsilon))(x) .
$$


The corresponding canonical scaling for the body forces writes

$$
f_{\alpha}(\varepsilon)(x):=f_{\alpha}^{\varepsilon}\left(x^{\varepsilon}\right) \quad \text { and } \quad f_{3}(\varepsilon)(x):=\varepsilon^{-1} f_{3}^{\varepsilon}\left(x^{\varepsilon}\right) .
$$

Our analysis holds if the scaled body force $f(\varepsilon)$ satisfies an asymptotic property like

$$
f(\varepsilon)(x) \simeq f^{0}(x)+\varepsilon f^{1}(x)+\varepsilon^{2} f^{2}(x)+\cdots+\varepsilon^{k} f^{k}(x)+\cdots
$$

But, for simplicity we assume here that the only coefficient $f_{0}$ is non zero. For example, if $f^{\varepsilon}$ is the force of gravity, this condition holds.

It may be directly seen that the scaled displacement $u(\varepsilon)$ solves the new problem:

$$
\begin{aligned}
u(\varepsilon) & \in V(\Omega), \\
\forall v & \in V(\Omega), \quad \int_{\Omega} A \kappa(\varepsilon)(u(\varepsilon)): \kappa(\varepsilon)(v)=\int_{\Omega} f \cdot v,
\end{aligned}
$$

where

$$
V(\Omega)=\left\{v \in H^{1}(\Omega)^{3} \mid \quad v=0 \quad \text { on } \quad \Gamma_{0}=\partial \omega \times(-1,1)\right\}
$$

and our purpose is to study the asymptotic behavior of $u(\varepsilon)$.

\subsection{Related questions}

There is a large amount of papers dealing with the asymptotic behavior of the displacement. We already mentioned [8] where the mixed variational formulation (involving both the stress tensor and the displacement) was used, rather than the "single" version that we use in this work, where the displacement remains the only unknown of the problem.

The model we develop here was extended to the case of coupled systems made of two different solids behaving in different ways at the limit as $\varepsilon \rightarrow 0$ : examples are a three-dimensional body in which a thin plate is inserted ( $c f$. [10]), or a thin plate where a slit contains a rod $(c f .[21])$, and also the junction between two plates $[25,27]$ or two rods ( $c f .[26])$. More recently, structures involving shells have been considered: then the geometry of the mean surface has to be taken into account; $c f .[11,12,13]$, [29], [35, 36, 37].

\subsection{Polynomial Ansätze and boundary layers}

The "asymptotic expansion method" for thin structures relies on special Ansätze about the behavior of $u(\varepsilon)$. 
(i) The polynomial Ansatz. We call polynomial Ansatz for $u(\varepsilon)$ a proposition of asymptotics of the form

$$
u(\varepsilon)(x) \simeq u^{0}(x)+\varepsilon u^{1}(x)+\varepsilon^{2} u^{2}(x)+\cdots+\varepsilon^{k} u^{k}(x)+\cdots
$$

where the functions $u^{0}, u^{1}$, etc... do not depend on $\varepsilon$. Inserting the Ansatz into the variational formulation (1.6) and identifying formally the powers of $\varepsilon$ in both sides of the equation, one obtains equations for $u^{0}, u^{1}$, etc... The hypothesis (1.3) leads to assume that the odd terms in (1.8a) do not appear. Thus, we arrive at the notion of "even polynomial Ansatz":

$$
u(\varepsilon)(x) \simeq u^{0}(x)+\varepsilon^{2} u^{2}(x)+\cdots+\varepsilon^{2 k} u^{2 k}(x)+\cdots
$$

In this respect, $[8,14]$ (see also [32]), have formally identified the three first terms of the Ansatz, namely $u^{0}, u^{2}$ and $u^{4}$.

(ii) Kirchhoff-Love Displacements. The first term $u^{0}$, as determined in the above mentioned works, satisfies the Dirichlet boundary conditions of the clamped plate and moreover

$$
e_{i 3}\left(u^{0}\right)=0 \quad \text { in } \quad \Omega, \quad i=1,2,3 .
$$

This is the Kirchhoff-Love hypothesis. Conditions (1.9) hold if and only if there exist 3 functions $\zeta_{1}^{0}, \zeta_{2}^{0}, \zeta_{3}^{0}$ defined on the two-dimensional mean surface $\omega$ such that

$$
u^{0}(x)=\left(\zeta_{1}^{0}\left(x_{1}, x_{2}\right)-x_{3} \partial_{1} \zeta_{3}^{0}\left(x_{1}, x_{2}\right), \zeta_{2}^{0}\left(x_{1}, x_{2}\right)-x_{3} \partial_{2} \zeta_{3}^{0}\left(x_{1}, x_{2}\right), \zeta_{3}^{0}\left(x_{1}, x_{2}\right)\right) .
$$

In other words $u^{0}$ is a Kirchhoff-Love displacement.

(iii) The Justification of the polynomial Ansatz. This is essential for the validation of the asymptotics. But neither $u^{2}$, nor $u^{4}$ in general, satisfies the Dirichlet boundary conditions. Such a situation contrasts with the case of periodic boundary conditions as investigated by J.C. PAUMIER in [34] where the validity and the convergence of the corresponding Ansatz (1.8b) are proved.

Thus, in the clamped plate, a method to perform such a justification is the construction of "correctors" as initiated by J.-L. Lions in [28]. By this strategy, P. Destuynder [14] and P. Destuynder \& I. Gruais [17] proved that the KirchhoffLove term $u^{0}$ is actually the limit of $u(\varepsilon)$ in the energy norm and that the following $H^{1}$ - estimates hold:

$$
\left\|u_{i}(\varepsilon)-u_{i}^{0}\right\|_{H^{1}(\Omega)} \leq C \varepsilon^{1 / 2} \quad i=1,2,3 .
$$

(iv) Boundary LAYERs. These estimates are limited to the first term $u^{0}$; this is linked to the fact that the other terms $u^{2}, u^{4}$, etc... do not satisfy the Dirichlet boundary conditions of the clamped plate. Even in this simplest case of the Dirichlet condition, the clamped part of the plate generates a "boundary layer" corresponding to an ill-posed problem. Typically, the elliptic problem (1.6) degenerates in the limit into a new problem which, roughly speaking, behaves like the Neumann problem for the operator $\partial_{3}^{2}$ and the surface $\Gamma^{0}$ is characteristic for this last operator. 
Generally speaking, such a phenomenon is classical for singular perturbation problems: see the book [24] by A. M. IL'IN, where the construction of matching outer and inner expansions (corresponding to the polynomial Ansatz and the boundary layer terms respectively) is explained on many examples.

(v) Multi-scale Asymptotics. The idea is to find profiles (see for instance the related works $[1,2]$ by G. Allaire and $[22,23]$ by O. GuÈs where this notion is currently used) $\Psi^{0}, \Psi^{1} \ldots$ which are functions of the space variable $x$ and also of other variables $T$ such that for a certain relation of dependence of $T$ with respect to $x$ and $\varepsilon, T=T(x, \varepsilon)$, we have

$$
u(\varepsilon)(x) \simeq \Psi^{0}(x, T)+\varepsilon \Psi^{1}(x, T)+\varepsilon^{2} \Psi^{2}(x, T)+\cdots+\varepsilon^{k} \Psi^{k}(x, T)+\cdots
$$

Such an approach is classical in homogeneization of periodic elastic structures: $c f$. the above references and the book by O.A. Oleinik, A.S. Shamaev \& G.A. Yosifian [33] and also the references therein.

For related problems in thin plates, we can cite D. N. Arnold \& R. S. FAlK [3] for the two-dimensional Reissner-Mindlin model and C. ScHwAB [39] for the heat equilibrium scalar equation. Finally, while writing this paper we found out that S. A. NAZAROV \& I. S. ZORIN [32] treated the same problem of thin plates for an isotropic material by this method of multi-scale asymptotics and determined the first terms up to the power $\varepsilon^{2}$.

In these two papers, our aim is threefold:

1. With the correct scaled variable(s) T, we construct the Ansatz (1.11) by calculating terms of arbitrarily high orders. This involves recurrence relations under the form of an algorithm providing the successive terms. The general setting of monoclinic materials allows a better understanding of the operators occurring in the algorithm.

2. We obtain optimal error estimates in the energy norm between the scaled displacement $u(\varepsilon)$ and a finite expansion where we keep only $N$ terms of the Ansatz (1.11). Such a strategy is original when compared with earlier work on this subject (at the exception of [32]).

3. We construct edge layer displacements along the edges $\partial \omega \times\{ \pm 1\}$ of $\Omega$ in order to obtain estimates in higher order norms and information about the structure of the solution.

\subsection{Outline of the paper and main results}

(i) Hypotheses. All our results are valid for the above assumptions about the rigidity matrix $A$, see (1.3), and the following smoothness hypotheses:

$$
\left\{\begin{array}{l}
\omega \text { has a } \mathscr{C}^{\infty} \text { boundary } \partial \omega \\
A=A\left(x_{1}, x_{2}\right) \in \mathscr{C}^{\infty}\left(\bar{\omega}, \mathscr{M}^{9 \times 9}\right) \\
f \in \mathscr{C}^{\infty}\left(\bar{\Omega}, \mathbb{R}^{3}\right)
\end{array}\right.
$$


These assumptions are necessary if one wants to push the asymptotics up to any order $N$ (see (1.13) below). But for any fixed $N$, it suffices that $f$ belongs to $H^{N+4}(\Omega)$ to obtain the estimates (1.14) below, see also in $\S 4.3$ the regularity of the terms in the asymptotics if $f$ is not supposed to be $\mathscr{C}^{\infty}$. On the other hand, the situation where the mean surface itself has corners ( $c f$. [31]), combined with jumps in the lateral boundary conditions, gives rise to different behaviors in the asymptotics.

(ii) Infinite ASYmptotic Expansion. We start with an even polynomial Ansatz of the type (1.8b). We prove in $\S 2$ (Theorem 2.4) that there exist unique terms $u^{0}, \ldots u^{2 k}, \ldots$ each one splitting into a function $v^{2 k}$ with zero mean values with respect to $x_{3}$ and into a Kirchhoff-Love displacement $u_{\mathrm{KL}}^{2 k}$ satisfying Dirichlet conditions on $\partial \omega$, such that the sum $\sum_{k} \varepsilon^{2 k} u^{2 k}$ is formally a solution of (1.6b). This construction relies on the solution of Neumann problems with respect to the variable $x_{3}$ on the interval $(-1,+1)$ and the difficulty is to fulfill the compatibility conditions on the right hand sides in each point of the mean surface $\omega$. With the exception of the first (Kirchhoff-Love) term $u^{0}$, the other terms do not satisfy the Dirichlet condition (1.6a).

Then we try to compensate for the Dirichlet boundary values of our even Ansatz by a boundary layer Ansatz of the form $\sum_{k \geq 1} \varepsilon^{k} w^{k}\left(\frac{r}{\varepsilon}, s, x_{3}\right)$ where $(r, s)$ is a system of coordinates in $\omega$ such that the equation of the boundary $\partial \omega$ is $r=0$. In order to reach this aim, considering the variable $s$ as a parameter, it suffices to work with operators in the normal variables $\left(t, x_{3}\right)=\left(\frac{r}{\varepsilon}, x_{3}\right)$ : in $\S 3$ we exhibit the corresponding reduction which consists of mixed Dirichlet-Neumann problems for a family of elasticity operators depending on the parameter $s$, called reduced-normal problems and defined on the half-strip

$$
\Sigma^{+}=\left\{\left(t, x_{3}\right) \in \mathbb{R}^{+} \times(-1,1)\right\}
$$

with non-homogeneous Dirichlet conditions on $\{0\} \times(-1,1)$ and zero Neumann conditions on the other sides $\mathbb{R}^{+} \times\{-1,1\}$. In this Part I, we only state the results which are needed here, and give the proofs in Part II. Roughly speaking, the solutions of these Dirichlet-Neumann problems are the sum of a rigid displacement (living in a space of dimension 4 ) and of a term exponentially decreasing at infinity.

Thus, at this stage, we have to correct the Dirichlet boundary values of our even Ansatz, in order that for each value of the parameter $s$, the "rigid displacement" is canceled. We perform in $\S 4$ such a correction by the help of an odd Ansatz. Then we achieve the construction of the whole asymptotics for $u(\varepsilon)$ by a two-step algorithm. The outcome of this algorithm (Theorem 4.1) is an asymptotics of the form (1.11)

$$
\begin{aligned}
& \Psi^{0}(x, t)=u_{\mathrm{KL}}^{0}(x), \quad \Psi^{1}(x, t)=u_{\mathrm{KL}}^{1}(x)-\chi(r) w^{1}\left(t, s, x_{3}\right) \\
& \Psi^{k}(x, t)=u_{\mathrm{KL}}^{k}(x)+v^{k}(x)-\chi(r) w^{k}\left(t, s, x_{3}\right) \text { for } k \geq 2 \\
& \text { with }\left\{\begin{array}{l}
u_{\mathrm{KL}}^{k} \text { a Kirchhoff-Love term, } \\
v^{k} \text { satisfies } \int_{-1}^{1} v^{k}\left(x_{1}, x_{2}, x_{3}\right) d x_{3}=0, \forall\left(x_{1}, x_{2}\right) \in \omega, \\
w^{k}\left(t, s, x_{3}\right) \text { is uniformly exponentially decreasing as } t \rightarrow+\infty, \\
\chi \text { is a cut-off function equal to } 1 \text { in a neighborhood of } r=0,
\end{array}\right.
\end{aligned}
$$


which is (formally) solution of the problem (1.6a) \& (1.6b). In this expansion, the "polynomial" part $\sum_{k \geq 0} \varepsilon^{k} u^{k}=\sum_{k \geq 0} \varepsilon^{k}\left(u_{\mathrm{KL}}^{k}+v^{k}\right)$ forms the polynomial Ansatz, whereas the part $\sum_{k \geq 1} \varepsilon^{k} w^{k}\left(\frac{r}{\varepsilon}, s, x_{3}\right)$ is the boundary layer Ansatz. Note that the boundary layer Ansatz starts with $\varepsilon$. It is interesting to compare the complexity of this algorithm, and in particular the occurrence of the odd polynomial terms due to the structure at infinity of solutions on the half strip $\Sigma^{+}$, with the situation of scalar second-order operators studied by C. SCHWAB [39]: in the scalar case, the interaction between polynomial Ansatz and boundary layer Ansatz is minimal (zero mean value for the Dirichlet condition in $t=0$ is sufficient to ensure the exponential decay for the boundary layer terms), all terms in the asymptotics are of even order and the terms in the polynomial Ansatz are explicit.

(iii) Error estimates for the Displacement field. Denoting for any $N \in \mathbb{N}$ by $U^{N}(\varepsilon)$ the truncated expansion (1.11):

$$
U^{N}(\varepsilon)(x)=\Psi^{0}\left(x, \frac{r}{\varepsilon}\right)+\varepsilon \Psi^{1}\left(x, \frac{r}{\varepsilon}\right)+\varepsilon^{2} \Psi^{2}\left(x, \frac{r}{\varepsilon}\right)+\cdots+\varepsilon^{N} \Psi^{N}\left(x, \frac{r}{\varepsilon}\right),
$$

we obtain in $\S 5$ optimal estimates of the error between $u(\varepsilon)$ and $U^{N}(\varepsilon)$ in energy norm. We use the classical method in asymptotic analysis consisting in pushing forward the asymptotics a few terms further (here $(N+4))$, deducing rough estimates by Korn's inequality and concluding by an estimate of the energy norm of the neglected terms $\left(\Psi^{N+1}, \Psi^{N+2}, \Psi^{N+3}, \Psi^{N+4}\right)$. Thus we prove in $\S 5$ that the error is of the same order as the energy norm of the first neglected term $\Psi^{N+1}$ :

$$
\left\|u(\varepsilon)-\sum_{k=0}^{N} \varepsilon^{k} u^{k}+\chi(r) \sum_{k=1}^{N} \varepsilon^{k} w^{k}\left(\frac{r}{\varepsilon}, s, x_{3}\right)\right\|_{H^{1}(\Omega)^{3}} \leq C \varepsilon^{N+1 / 2},
$$

and if one adds the boundary layer term of order $N+1$

$$
\left\|u(\varepsilon)-\sum_{k=0}^{N} \varepsilon^{k} u^{k}+\chi(r) \sum_{k=1}^{N+1} \varepsilon^{k} w^{k}\left(\frac{r}{\varepsilon}, s, x_{3}\right)\right\|_{H^{1}(\Omega)^{3}} \leq C \varepsilon^{N+1} .
$$

We moreover give estimates in $L^{2}$-norm:

$$
\left\|u(\varepsilon)-\sum_{k=0}^{N} \varepsilon^{k} u^{k}+\chi(r) \sum_{k=1}^{N} \varepsilon^{k} w^{k}\left(\frac{r}{\varepsilon}, s, x_{3}\right)\right\|_{L^{2}(\Omega)^{3}} \leq C \varepsilon^{N+1},
$$

and if one adds the polynomial term of order $N+1$

$$
\left\|u(\varepsilon)-\sum_{k=0}^{N+1} \varepsilon^{k} u^{k}+\chi(r) \sum_{k=1}^{N} \varepsilon^{k} w^{k}\left(\frac{r}{\varepsilon}, s, x_{3}\right)\right\|_{H^{1}(\Omega)^{3}} \leq C \varepsilon^{N+3 / 2} .
$$

(iv) Relation With the Kirchhoff-Love Model AND Other Models of PLATES. The first terms in the asymptotics have a special form. At the zero order, we recover the well-known fact that the first approximation for $u(\varepsilon)$ is $U^{0}=u_{\mathrm{KL}}^{0}$ :

$$
u_{\mathrm{KL}}^{0}(x)=\left(\zeta_{1}^{0}-x_{3} \partial_{1} \zeta_{3}^{0}, \zeta_{2}^{0}-x_{3} \partial_{2} \zeta_{3}^{0}, \zeta_{3}^{0}\right),
$$


with $\left(\zeta_{1}^{0}, \zeta_{2}^{0}\right)=\zeta_{*}^{0}$ the unique solution on the mean surface $\omega$ of the membrane equation

$$
\zeta_{*}^{0} \in \stackrel{\circ}{H}^{1}(\omega)^{2} \quad \text { and } \quad-\partial_{\beta} M_{\alpha \beta \gamma \delta} e_{\gamma \delta}\left(\zeta_{*}^{0}\right)=\frac{1}{2} \int_{-1}^{+1} f_{\alpha}^{0} d x_{3},
$$

associated to the resultant rigidity matrix (which is positive definite)

$$
M_{\alpha \beta \gamma \delta}=A_{\alpha \beta \gamma \delta}-A_{\alpha \beta 33} A_{3333}^{-1} A_{33 \gamma \delta},
$$

and $\zeta_{3}^{0}$ is the solution of the bending equation

$$
\zeta_{3}^{0} \in \stackrel{\circ}{H}^{2}(\omega) \quad \text { and } \quad \partial_{\alpha \beta} M_{\alpha \beta \gamma \delta} \partial_{\gamma \delta}\left(\zeta_{3}^{0}\right)=\frac{3}{2} \int_{-1}^{+1}\left(f_{3}^{0}+x_{3} \partial_{\alpha} f_{\alpha}^{0}\right) d x_{3}
$$

associated with the same rigidity matrix $M$. In the case of an isotropic material of Lamé constants $\lambda$ and $\mu$,

$$
A_{i j k l}=\lambda \delta_{i j} \delta_{k l}+\mu\left(\delta_{i k} \delta_{j l}+\delta_{i l} \delta_{j k}\right),
$$

and:

$$
M_{\alpha \beta \gamma \delta}=\frac{2 \lambda \mu}{\lambda+2 \mu} \delta_{\alpha \beta} \delta_{\gamma \delta}+\mu\left(\delta_{\alpha \gamma} \delta_{\beta \delta}+\delta_{\alpha \delta} \delta_{\beta \gamma}\right)
$$

At the order one, $u^{1}$ coincides with the Kirchhoff-Love displacement $u_{\mathrm{KL}}^{1}$, whose "generating functions" $\left(\zeta_{1}^{1}, \zeta_{2}^{1}\right)$ and $\zeta_{3}^{1}$ are solutions of a membrane equation and a bending equation respectively, with zero right-hand side and a Dirichlet condition equal to the traces of the rigid displacements appearing for each $s$ in the behavior at infinity of the solution of the "reduced-normal problem" with Dirichlet data equal to the traces of $v^{2} c f$. (4.4) and (4.5). We will prove in Part II that the function $u_{\mathrm{KL}}^{1}$ is not zero in general. The first term in the boundary layer series correlatively appears: this is $w^{1}$ and its third component $w_{3}^{1}$ is zero.

Taking into account the form of the first terms of the asymptotics we obtain the following error estimates between $u(\varepsilon)$ and $u_{\mathrm{KL}}^{0}$ :

$$
\begin{cases}\left\|u_{\alpha}(\varepsilon)-u_{\mathrm{KL}, \alpha}^{0}(\varepsilon)\right\|_{H^{1}(\Omega)} \leq C \varepsilon^{1 / 2} & \left\|u_{3}(\varepsilon)-u_{\mathrm{KL}, 3}^{0}(\varepsilon)\right\|_{H^{1}(\Omega)} \leq C \varepsilon \\ \left\|u_{\alpha}(\varepsilon)-u_{\mathrm{KL}, \alpha}^{0}(\varepsilon)\right\|_{L^{2}(\Omega)} \leq C \varepsilon & \left\|u_{3}(\varepsilon)-u_{\mathrm{KL}, 3}^{0}(\varepsilon)\right\|_{L^{2}(\Omega)} \leq C \varepsilon .\end{cases}
$$

Moreover, the estimates (1.14) allow a precise evaluation of the approximation of the true displacement $u(\varepsilon)$ by a Kirchhoff-Love displacement $u_{\mathrm{KL}}(\varepsilon)$ : let us take

$$
u_{\mathrm{KL}}(\varepsilon)=u_{\mathrm{KL}}^{0}+\varepsilon u_{\mathrm{KL}}^{1} .
$$

Then (1.14a)-(1.14d) yield the estimates

$$
\begin{cases}\left\|u_{\alpha}(\varepsilon)-u_{\mathrm{KL}, \alpha}(\varepsilon)\right\|_{H^{1}(\Omega)} \leq C \varepsilon^{1 / 2} & \left\|u_{3}(\varepsilon)-u_{\mathrm{KL}, 3}(\varepsilon)\right\|_{H^{1}(\Omega)} \leq C \varepsilon^{3 / 2}, \\ \left\|u_{\alpha}(\varepsilon)-u_{\mathrm{KL}, \alpha}(\varepsilon)\right\|_{L^{2}(\Omega)} \leq C \varepsilon^{3 / 2} & \left\|u_{3}(\varepsilon)-u_{\mathrm{KL}, 3}(\varepsilon)\right\|_{L^{2}(\Omega)} \leq C \varepsilon^{2}\end{cases}
$$


Only the estimate on the first two components in $H^{1}$-norm is not improved by the adjunction of $\varepsilon u_{\mathrm{KL}}^{1}$. We will not obtain any improvement of the three other estimates if we add $\varepsilon^{2} u_{\mathrm{KL}}^{2}$ to $u_{\mathrm{KL}}(\varepsilon)$. But, if we consider now the scaled linearized strain tensor, we have to include the polynomial terms up to the order 2 to obtain a convergence:

$$
\left\|\kappa(\varepsilon)\left(u(\varepsilon)-\left(u_{\mathrm{KL}}^{0}+\varepsilon u_{\mathrm{KL}}^{1}+\varepsilon^{2} u_{\mathrm{KL}}^{2}+v^{2}\right)\right)\right\|_{L^{2}(\Omega)^{9}} \leq C \varepsilon^{1 / 2} .
$$

We note that, with only one exception, all the above estimates are limited by the boundary layer terms. Since the boundary layer terms are not polynomial in the variable $x_{3}$ in general, other bi-dimensional models of plates (for example ReissnerMindlin, or more generally Naghdi plates theories [30] or standard polynomial hierarchical models of plates) seem to be unable to provide a better approximation in energy norm as $\varepsilon \rightarrow 0$ than the Kirchhoff-Love model.

On the other hand, the third component $u_{3}(\varepsilon)$ of the displacement would be better described in the norm $L^{2}(\Omega)$ by a model which could be able to include

$v_{3}^{2}$. In the same order of idea, if one evaluates of the error in other norms such as $H^{1}\left((-1,+1), H^{-1}(\omega)\right)$ or $H^{2}\left((-1,+1), H^{-2}(\omega)\right)$, or considers some average norms, higher order models can be more performant. An interesting application of this work could be a more precise investigation of higher order models of plates: $c f$. [4] for the analysis of shear correction factors and [38] for a-posteriori estimates.

\section{THE "EVEN" ANSATZ}

In this section, we are going to present an algorithm for the construction of an infinite even polynomial Ansatz of the type (1.8b) for the solution of problem (1.6). Our algorithm is an extension of the calculations of [8, 14], [32] determining the first two or three terms of this Ansatz.

\subsection{The sequence of boundary value problems}

Let us recall that problem (1.6) is a mixed boundary value problem with homogeneous Dirichlet conditions on $\Gamma_{0}=\partial \omega \times(-1,1)$. For the presentation of our algorithm, it is easier to consider non-homogeneous Dirichlet conditions and volume forces set to 0 . We return to the original problem (1.6) at the end of this section (§2.4). Let there be given $h$ a triple of traces $h=\left(h_{1}, h_{2}, h_{3}\right)$ in $\mathscr{C}^{\infty}\left(\bar{\Gamma}_{0}\right)$. The problem we now consider is:

$$
\begin{aligned}
& u(\varepsilon) \in H^{1}(\Omega)^{3} \quad \text { and } \quad u(\varepsilon)=h \text { on } \Gamma_{0}, \\
& \forall v \in V(\Omega), \quad \int_{\Omega} A \kappa(\varepsilon)(u(\varepsilon)): \kappa(\varepsilon)(v)=0 .
\end{aligned}
$$

Moreover, problem (2.1) will be useful by itself in further developments $(\S 4)$. 
Using the definition (1.4a) of $\kappa(\varepsilon)$ and integrating by parts, we obtain the following equivalent form for problem (2.1), which we split into the two groups of equations (2.2) and (2.3):

$$
\begin{aligned}
& u_{3}(\varepsilon)=h_{3} \text { on } \Gamma_{0}, \\
& A_{3333} \partial_{33} u_{3}(\varepsilon)+\varepsilon^{2}\left(A_{33 \gamma \delta} \partial_{3} e_{\gamma \delta}(u(\varepsilon))+2 \partial_{\alpha} A_{\alpha 3 \gamma 3} e_{\gamma 3}(u(\varepsilon))\right)=0 \text { in } \Omega, \\
& A_{3333} \partial_{3} u_{3}(\varepsilon)+\varepsilon^{2} A_{33 \gamma \delta} e_{\gamma \delta}(u(\varepsilon))=0 \text { on }\left\{x_{3}= \pm 1\right\} \cap \partial \Omega, \\
& u_{\alpha}(\varepsilon)=h_{\alpha} \text { on } \Gamma_{0}, \\
& 2 A_{\alpha 3 \gamma 3} \partial_{3} e_{\gamma 3}(u(\varepsilon))+\partial_{\beta} A_{\alpha \beta 33} \partial_{3} u_{3}(\varepsilon)+\varepsilon^{2} \partial_{\beta} A_{\alpha \beta \gamma \delta} e_{\gamma \delta}(u(\varepsilon))=0 \text { in } \Omega, \\
& A_{\alpha 3 \gamma 3} e_{\gamma 3}(u(\varepsilon))=0 \text { on }\left\{x_{3}= \pm 1\right\} \cap \partial \Omega
\end{aligned}
$$

We use the repeated index convention along with the notation which gives to the Greek indices $\alpha, \beta, \gamma, \ldots$ the values in $\{1,2\}$ corresponding to the in-plane variables. In this calculation, we took into account the fact that the coefficients of the rigidity matrix may depend on $\left(x_{1}, x_{2}\right)$ but not on $x_{3}$. Equations (2.2a) and (2.3a) are the Dirichlet conditions on $u_{3}$ and $\left(u_{1}, u_{2}\right)$ respectively, $(2.2 \mathrm{c})$ and $(2.3 \mathrm{c})$ the Neumann conditions, and $(2.2 \mathrm{~b})$ and $(2.3 \mathrm{~b})$ the interior equations.

As a consequence of hypothesis (1.3) about the coefficients of the rigidity matrix $A$, the only powers of $\varepsilon$ present in (2.2)-(2.3) are $\varepsilon^{0}$ and $\varepsilon^{2}$. This is the reason for the introduction of the even Ansatz $u(\varepsilon)(x) \simeq u^{0}(x)+\varepsilon^{2} u^{2}(x)+\cdots+\varepsilon^{2 k} u^{2 k}(x)+\cdots$ Inserting this Ansatz in equations of (2.2) and (2.3) and identifying to 0 the factors of all the powers of $\varepsilon$, we find for any $k \in \mathbb{N}$ :

$$
\begin{aligned}
& u_{3}^{2 k}=h_{3}^{2 k} \text { on } \Gamma_{0}, \\
& A_{3333} \partial_{33} u_{3}^{2 k}+A_{33 \gamma \delta} \partial_{3} e_{\gamma \delta}\left(u^{2 k-2}\right)+2 \partial_{\alpha} A_{\alpha 3 \gamma 3} e_{\gamma 3}\left(u^{2 k-2}\right)=0 \text { in } \Omega, \\
& A_{3333} \partial_{3} u_{3}^{2 k}+A_{33 \gamma \delta} e_{\gamma \delta}\left(u^{2 k-2}\right)=0 \text { on }\left\{x_{3}= \pm 1\right\} \cap \partial \Omega, \\
& u_{\alpha}^{2 k}=h_{\alpha}^{2 k} \text { on } \Gamma_{0}, \\
& 2 A_{\alpha 3 \gamma 3} \partial_{3} e_{\gamma 3}\left(u^{2 k}\right)+\partial_{\beta} A_{\alpha \beta 33} \partial_{3} u_{3}^{2 k}+\partial_{\beta} A_{\alpha \beta \gamma \delta} e_{\gamma \delta}\left(u^{2 k-2}\right)=0 \text { in } \Omega, \\
& A_{\alpha 3 \gamma 3} e_{\gamma 3}\left(u^{2 k}\right)=0 \text { on }\left\{x_{3}= \pm 1\right\} \cap \partial \Omega
\end{aligned}
$$

where we have set $h^{2 k}=0$ for $k>0$ and $u^{2 k-2}=0$ for $k=0$.

\section{$2.2 \quad$ Kirchhoff-Love displacements}

For $k=0$, these two systems reduce to

$$
\begin{aligned}
u_{3}^{0}=h_{3}^{0} \quad & \text { on } \quad \Gamma_{0}, \\
A_{3333} \partial_{33} u_{3}^{0}=0 & \text { in } \quad \Omega, \\
A_{3333} \partial_{3} u_{3}^{0}=0 & \text { on } \quad\left\{x_{3}= \pm 1\right\} \cap \partial \Omega,
\end{aligned}
$$




$$
\begin{aligned}
u_{\alpha}^{0} & =h_{\alpha}^{0} & & \text { on } \quad \Gamma_{0}, \\
2 A_{\alpha 3 \gamma 3} \partial_{3} e_{\gamma 3}\left(u^{0}\right)+\partial_{\beta} A_{\alpha \beta 33} \partial_{3} u_{3}^{0} & =0 & & \text { in } \quad \Omega, \\
A_{\alpha 3 \gamma 3} e_{\gamma 3}\left(u^{0}\right) & =0 & & \text { on } \quad\left\{x_{3}= \pm 1\right\} \cap \partial \Omega .
\end{aligned}
$$

To solve problems (2.6) and (2.7) (and more generally problems (2.4) and (2.5)), we note that, since $A$ is positive definite, its diagonal elements are $>0$ and its diagonal blocks are positive definite. Whence

$$
A_{3333}>0 \text { and }\left(A_{\alpha 3 \gamma 3}\right)=\left(\begin{array}{ll}
A_{1313} & A_{1323} \\
A_{2313} & A_{2323}
\end{array}\right) \text { is positive definite. }
$$

As a consequence of (2.8) we find that the only solutions of (2.6b)-(2.6c) are the functions $u_{3}^{0}\left(x_{*}, x_{3}\right)=\zeta_{3}^{0}\left(x_{*}\right)$ which do not depend on $x_{3}$. Here and elsewhere, $x_{*}$ denotes the couple $\left(x_{1}, x_{2}\right)$ of variables in the mean surface $\omega$. Therefore, a necessary and sufficient condition to ensure the Dirichlet condition (2.6a) is that $h_{3}^{0}$ does not depend on $x_{3}$.

Taking advantage of (2.8) again, we find that problem (2.7) reduces to the following system whose unknown is $u_{*}=\left(u_{1}, u_{2}\right)$ (with the same convention of notation $\nabla_{*}$ denotes the gradient $\left(\partial_{1}, \partial_{2}\right)$ in $\left.\omega\right)$ :

$$
\begin{aligned}
u_{*}^{0}=h_{*}^{0} \quad & \text { on } \quad \Gamma_{0}, \\
\partial_{33} u_{*}^{0}=0 & \text { in } \quad \Omega, \\
\partial_{3} u_{*}^{0}+\nabla_{*} \zeta_{3}^{0}=0 \quad & \text { on } \quad\left\{x_{3}= \pm 1\right\} \cap \partial \Omega .
\end{aligned}
$$

Thus the general solution of $(2.9 \mathrm{~b})-(2.9 \mathrm{c})$ is $u_{*}^{0}=\zeta_{*}^{0}-x_{3} \nabla_{*} \zeta_{3}^{0}$, where $\zeta_{*}^{0}=\left(\zeta_{1}^{0}, \zeta_{2}^{0}\right)$ does not depend on $x_{3}$. In concordance with [7, 11.4$]$, we may set:

Definition 2.1 We call Kirchhoff-Love displacement on $\Omega$ and denote by $u_{\mathrm{KL}}$ any displacement of the form

$$
u_{\mathrm{KL}}=\left(u_{\mathrm{KL}, *}, u_{\mathrm{KL}, 3}\right) \quad \text { with } \quad u_{\mathrm{KL}, 3}=\zeta_{3} \quad \text { and } \quad u_{\mathrm{KL}, *}=\zeta_{*}-x_{3} \nabla_{*} \zeta_{3}
$$

where $\zeta_{3}$ and $\zeta_{*}$ are functions of $x_{*}$ defined on $\bar{\omega}$, called the generating functions of $u_{\mathrm{KL}}$. As a consequence of this definition $e_{i 3}\left(u_{\mathrm{KL}}\right)=0$.

A necessary condition to ensure the Dirichlet condition (2.9a) is that $h_{*}^{0}$ be an affine function of $x_{3}$ for any $x_{*} \in \partial \omega$. Let us investigate the sufficiency.

As already hinted in $\S 1$, we introduce smooth local coordinates $(r, s)$ in a tubular neighborhood $\mathcal{V}$ of $\partial \omega$. Let $L_{1}, \ldots, L_{I}$ be the lengths of the connected components of $\partial \omega$. We denote by $S$ the disjoint union

$$
S=L_{1} \mathbb{S}^{1} \dot{\cup} \cdots \dot{\cup} L_{I} \mathbb{S}^{1} .
$$


The variable $r$ belongs to $(-\rho, \rho)$ and behaves like the distance to the boundary $\partial \omega$ inside $\omega$ and $s$ belongs to $S$ and behaves like the arc length along $\partial \omega$ :

$$
\text { for } x_{*}=X_{*}(r, s) \quad\left\{\begin{array}{l}
x_{*} \in \mathcal{V} \cap \omega \Longleftrightarrow 0<r<\rho, s \in S \\
x_{*} \in \partial \omega \Longleftrightarrow r=0, s \in S, \\
x_{*} \in \partial \omega \Longrightarrow D X_{*}\left(x_{*}\right) \in \mathrm{SO} 2 .
\end{array}\right.
$$

Correspondingly, we define the contravariant change of coordinates on a displacement field $u_{*}$ defined on $\bar{\omega}$, by

$$
u_{*}=u_{1} e_{1}+u_{2} e_{2}=u_{r} n_{r}+u_{s} n_{s}
$$

where $\left(e_{1}, e_{2}\right)$ is the Cartesian basis, and

$$
n_{r}(r, s)=D X_{*}\left(x_{*}\right) e_{1}, \quad n_{s}(r, s)=D X_{*}\left(x_{*}\right) e_{2}
$$

whence

$$
u_{r}(r, s)=D X_{*}\left(x_{*}\right)^{-1} u_{*}\left(x_{*}\right) \cdot e_{1}, \quad u_{s}(r, s)=D X_{*}\left(x_{*}\right)^{-1} u_{*}\left(x_{*}\right) \cdot e_{2} .
$$

Then we see that for any $x_{*} \in \partial \omega$ :

$$
\zeta_{*}^{0}\left(x_{*}\right)-x_{3} \nabla_{*} \zeta_{3}^{0}\left(x_{*}\right)=\left(\zeta_{r}^{0}(0, s)-x_{3} \partial_{r} \zeta_{3}^{0}(0, s)\right) n_{r}+\left(\zeta_{s}^{0}(0, s)-x_{3} \partial_{s} \zeta_{3}^{0}(0, s)\right) n_{s}
$$

whence the Lemma:

Lemma 2.2 Let $h_{r}, h_{n}, h_{s}$ and $h_{3}$ be four smooth functions in $\mathscr{C}^{\infty}(\partial \omega)$. Then problem (2.6)-(2.7) with the Dirichlet data

$$
h_{*}^{0}=\left(h_{r}-x_{3} h_{n}\right) n_{r}+\left(h_{s}-x_{3} \partial_{s} h_{3}\right) n_{s} \quad \text { and } \quad h_{3}^{0}=h_{3}
$$

has solutions which are Kirchhoff-Love displacements $u_{\mathrm{KL}}^{0}$. The functions $\zeta_{3}^{0}$ and $\zeta_{*}^{0}$ such that $u_{\mathrm{KL}, 3}^{0}=\zeta_{3}^{0}$ and $u_{\mathrm{KL}, *}^{0}=\zeta_{*}^{0}-x_{3} \nabla_{*} \zeta_{3}^{0}$ satisfy

$$
\zeta_{3}^{0}(0, s)=h_{3}(s), \quad \partial_{r} \zeta_{3}^{0}(0, s)=h_{n}(s), \quad \zeta_{r}^{0}(0, s)=h_{r}(s), \quad \zeta_{s}^{0}(0, s)=h_{s}(s)
$$

At this stage, the generating functions $\zeta_{3}^{0}$ and $\zeta_{*}^{0}$ are not uniquely determined. But, as we are going to show, if we solve problem (2.4) for $k=1,2$ and problem (2.5) for $k=1$, we arrive at the equation of a membrane for $\zeta_{*}^{0}$ and an equation of bending for $\zeta_{3}^{0}$, and then these functions are uniquely determined.

We can also see that the conditions for solving a Dirichlet problem are very restrictive. But, in particular, if we consider homogeneous Dirichlet conditions, we have the solvability. Hence, there is no boundary layer at the rank of $\varepsilon^{0}$. 


\subsection{Existence and uniqueness of an even Ansatz}

We are ready to prove by induction the following theorem:

Theorem 2.3 Let for any $k \in \mathbb{N}$, four functions $h_{r}^{2 k}, h_{n}^{2 k}, h_{s}^{2 k}$ and $h_{3}^{2 k}$ be given in $\mathscr{C}^{\infty}(\partial \omega)$. Moreover, we set $u^{-2}=0$. Then, there exist a unique sequence of displacements $\left(v^{2 k}\right)_{k \in \mathbb{N}}$ and a unique sequence of Kirchhoff-Love displacements $\left(u_{\mathrm{KL}}^{2 k}\right)_{k \in \mathbb{N}}$ such that for any $k \in \mathbb{N}$

$$
\left\{\begin{array}{l}
u^{2 k}:=v^{2 k}+u_{\mathrm{KL}}^{2 k} \text { is solution of }(2.4 \mathrm{~b})-(2.4 \mathrm{c}) \&(2.5 \mathrm{~b})-(2.5 \mathrm{c}), \\
\forall x_{*} \in \bar{\omega}, \quad \int_{-1}^{+1} v^{2 k}\left(x_{*}, x_{3}\right) d x_{3}=0, \\
u_{\mathrm{KL}}^{2 k}=\left(\zeta_{*}^{2 k}-x_{3} \nabla_{*} \zeta_{3}^{2 k}, \zeta_{3}^{2 k}\right) \quad \text { and } \\
\left.\quad \zeta_{3}^{2 k}\right|_{\partial \omega}=h_{3}^{2 k},\left.\quad \partial_{r} \zeta_{3}^{2 k}\right|_{\partial \omega}=h_{n}^{2 k},\left.\quad \zeta_{r}^{2 k}\right|_{\partial \omega}=h_{r}^{2 k},\left.\quad \zeta_{s}^{2 k}\right|_{\partial \omega}=h_{s}^{2 k} .
\end{array}\right.
$$

The functions $v^{2 k}$ and $u_{\mathrm{KL}}^{2 k}$ are smooth on $\bar{\Omega}$.

Proof. If $u^{2 k-2}$ is given, problem (2.4b)-(2.4c) is simply a Neumann problem on the interval $(-1,+1)$ for each fixed $x_{*} \in \bar{\omega}$. Thus the solvability of this problem relies upon a compatibility condition obtained by integrating $(2.4 \mathrm{~b})$ with respect to $x_{3}$ from -1 to +1 for any fixed $x_{*} \in \bar{\omega}$ and using the Neumann boundary conditions (2.4c). We obtain the system:

$$
\begin{aligned}
& 2 \partial_{\alpha} A_{\alpha 3 \gamma 3} \int_{-1}^{+1} e_{\gamma 3}\left(u^{2 k-2}\right) d x_{3}=0 \\
& A_{3333} \partial_{33} u_{3}^{2 k}=-A_{33 \gamma \delta} \partial_{3} e_{\gamma \delta}\left(u^{2 k-2}\right)-2 \partial_{\alpha} A_{\alpha 3 \gamma 3} e_{\gamma 3}\left(u^{2 k-2}\right) \text { in } \Omega, \\
& A_{3333} \partial_{3} u_{3}^{2 k}=-A_{33 \gamma \delta} e_{\gamma \delta}\left(u^{2 k-2}\right) \text { on }\left\{x_{3}= \pm 1\right\} \cap \partial \Omega .
\end{aligned}
$$

Taking advantage of (2.8), if $u_{3}^{2 k}$ is given, problem $(2.5 \mathrm{~b})-(2.5 \mathrm{c})$ is also a Neumann problem and its solvability relies upon the compatibility condition obtained by integrating $(2.5 \mathrm{~b})$ with respect to $x_{3}$ from -1 to +1 and using the Neumann boundary conditions $(2.5 \mathrm{c})$. We obtain the system:

$$
\begin{aligned}
& \partial_{\beta} A_{\alpha \beta 33} \int_{-1}^{+1} \partial_{3} u_{3}^{2 k} d x_{3}+\partial_{\beta} A_{\alpha \beta \gamma \delta} \int_{-1}^{+1} e_{\gamma \delta}\left(u^{2 k-2}\right) d x_{3}=0 \\
& A_{\alpha 3 \gamma 3} \partial_{33} u_{\gamma}^{2 k}=-A_{\alpha 3 \gamma 3} \partial_{3 \gamma} u_{3}^{2 k}-\partial_{\beta} A_{\alpha \beta 33} \partial_{3} u_{3}^{2 k}-\partial_{\beta} A_{\alpha \beta \gamma \delta} e_{\gamma \delta}\left(u^{2 k-2}\right) \text { in } \Omega \\
& A_{\alpha 3 \gamma 3} \partial_{3} u_{\gamma}^{2 k}=-A_{\alpha 3 \gamma 3} \partial_{\gamma} u_{3}^{2 k}, \quad \text { on }\left\{x_{3}= \pm 1\right\} \cap \partial \Omega .
\end{aligned}
$$

Let us formulate now our induction hypothesis for any $\ell \in \mathbb{N}$ :

$$
\left(\mathscr{I}^{\ell}\right) \quad \begin{cases}\forall k \leq \ell-1, & u^{2 k} \text { is determined and pb (2.15) \& (2.16) solved, } \\ v^{2 \ell} \text { is s.t. } & \forall x_{*} \in \bar{\omega}, \quad \int_{-1}^{+1} v^{2 \ell}\left(x_{*}, x_{3}\right) d x_{3}=0 \\ & (2.15) \&(2.16) \text { hold for } k=\ell \text { with } u^{2 \ell}=v^{2 \ell} \\ & \text { (2.15a) holds for } k=\ell+1 \text { with } u^{2 \ell}=v^{2 \ell} .\end{cases}
$$


It is clear that $\left(\mathscr{I}^{0}\right)$ holds with $u^{2 k}=0$ for $k<0$ and $v^{0}=0$.

Let us assume that $\left(\mathscr{I}^{\ell}\right)$ holds. Thus $v_{3}^{2 \ell}$ is a solution of $(2.15 \mathrm{~b})-(2.15 \mathrm{c})$. Therefore, for any function $\zeta_{3}^{2 \ell} \in \mathscr{C}^{\infty}(\bar{\omega})$,

$$
v_{3}^{2 \ell}+\zeta_{3}^{2 \ell} \text { is a solution of }(2.15 \mathrm{~b})-(2.15 \mathrm{c}) \text {. }
$$

Since $v_{*}^{2 \ell}$ is a solution of $(2.16 \mathrm{~b})-(2.16 \mathrm{c})$ with $u_{3}^{2 \ell}=v_{3}^{2 \ell}$, we easily see that for any function $\zeta_{*}^{2 \ell} \in \mathscr{C}^{\infty}(\bar{\omega})^{2}$,

$$
v_{*}^{2 \ell}+\zeta_{*}^{2 \ell}-x_{3} \nabla_{*} \zeta_{3}^{2 \ell} \text { is a solution of }(2.16 \mathrm{~b})-(2.16 \mathrm{c}) \text { with } u_{3}^{2 \ell}=v_{3}^{2 \ell}+\zeta_{3}^{2 \ell} .
$$

In other words, for any Kirchhoff-Love displacement $u_{\mathrm{KL}}^{2 \ell}$, the function $u^{2 \ell}=v^{2 \ell}+u_{\mathrm{KL}}^{2 \ell}$ solves (2.15) and (2.16) for $k=\ell$. Moreover, since (2.15a) holds for $k=\ell+1$ with $u^{2 \ell}=v^{2 \ell}$, it is straightforward that (2.15a) still holds for $k=\ell+1$ with $u^{2 \ell}=v^{2 \ell}+u_{\mathrm{KL}}^{2 \ell}$. As $A_{3333} \neq 0$, for any Kirchhoff-Love displacement $u_{\mathrm{KL}}^{2 \ell}$, there exists a unique function $v_{3}^{2 \ell+2}$ with zero mean values over $x_{3}$ which solves problem (2.15) for $k=\ell+1$ with $u^{2 \ell}=v^{2 \ell}+u_{\mathrm{KL}}^{2 \ell}$.

Now we investigate condition (2.16a) for $k=\ell+1$. We "eliminate" the term $\partial_{\beta} A_{\alpha \beta 33} \int_{-1}^{+1} \partial_{3} u_{3}^{2 \ell+2} d x_{3}$ with the help of problem (2.15) at the step $k=\ell+1$ : integrating (2.15b) in $x_{3}$ from the limit -1 and taking advantage of $(2.15 \mathrm{c})$ we find that

$$
A_{3333} \partial_{3} u_{3}^{2 \ell+2}=-A_{33 \gamma \delta} e_{\gamma \delta}\left(u_{*}^{2 \ell}\right)-2 \partial_{\alpha} A_{\alpha 3 \gamma 3} \int_{-1}^{x_{3}} e_{\gamma 3}\left(u^{2 \ell}\right) .
$$

We examine the contributions of $v^{2 \ell}$ and $u_{\mathrm{KL}}^{2 \ell}$. Since $e_{\gamma 3}\left(u_{\mathrm{KL}}^{2 \ell}\right)=0$, we obtain:

$A_{3333} \partial_{3} u_{3}^{2 \ell+2}=-A_{33 \gamma \delta} e_{\gamma \delta}\left(\zeta_{*}^{2 \ell}-x_{3} \nabla_{*} \zeta_{3}^{2 \ell}\right)-A_{33 \gamma \delta} e_{\gamma \delta}\left(v_{*}^{2 \ell}\right)-2 \partial_{\alpha} A_{\alpha 3 \gamma 3} \int_{-1}^{x_{3}} e_{\gamma 3}\left(v^{2 \ell}\right)$.

Whence, taking into account that the mean value of $e_{\gamma \delta}\left(x_{3} \nabla_{*} \zeta_{3}^{2 \ell}\right)$ is zero, the expression for condition (2.16a) when $k=\ell+1$ :

$$
\begin{aligned}
-\partial_{\beta} A_{\alpha \beta 33} A_{3333}^{-1} \int_{-1}^{+1} & \left(A_{33 \gamma \delta} e_{\gamma \delta}\left(\zeta_{*}^{2 \ell}\right)+A_{33 \gamma \delta} e_{\gamma \delta}\left(v_{*}^{2 \ell}\right)+2 \partial_{\alpha} A_{\alpha 3 \gamma 3} \int_{-1}^{x_{3}} e_{\gamma 3}\left(v^{2 \ell}\right)\right) d x_{3} \\
& +\partial_{\beta} A_{\alpha \beta \gamma \delta} \int_{-1}^{+1} e_{\gamma \delta}\left(\zeta_{*}^{2 \ell}\right) d x_{3}+\partial_{\beta} A_{\alpha \beta \gamma \delta} \int_{-1}^{+1} e_{\gamma \delta}\left(v_{*}^{2 \ell}\right) d x_{3}=0 .
\end{aligned}
$$

We put together the terms which depend only on $v^{2 \ell}$ and, taking advantage of the fact that $\int_{-1}^{+1} v^{2 \ell} d x_{3}=0$ we set:

$$
F_{\alpha}\left(v^{2 \ell}\right)=\partial_{\beta} A_{\alpha \beta 33} A_{3333}^{-1} \int_{-1}^{+1}\left(2 \partial_{\alpha} A_{\alpha 3 \gamma 3} \int_{-1}^{x_{3}} e_{\gamma 3}\left(v^{2 \ell}\right)\right) d x_{3} .
$$

Noting that the integrands involving $\zeta_{*}^{2 \ell}$ do not depend on $x_{3}$, our condition (2.16a) when $k=\ell+1$ takes the form:

$$
-\partial_{\beta} A_{\alpha \beta 33} A_{3333}^{-1} A_{33 \gamma \delta} e_{\gamma \delta}\left(\zeta_{*}^{2 \ell}\right)+\partial_{\beta} A_{\alpha \beta \gamma \delta} e_{\gamma \delta}\left(\zeta_{*}^{2 \ell}\right)=\frac{1}{2} F_{\alpha}\left(v^{2 \ell}\right) .
$$

But, as a consequence of the positivity of $A$, (see D. CAILlerie [5]), the $4 \times 4$ matrix:

$$
M_{\alpha \beta \gamma \delta}=A_{\alpha \beta \gamma \delta}-A_{\alpha \beta 33} A_{3333}^{-1} A_{33 \gamma \delta} \text { is positive definite. }
$$


Thus (2.17) is the equation of a membrane in the mean surface $\omega$ associated with the new two-dimensional "homogenized" rigidity matrix $M_{\alpha \beta \gamma \delta}$. We can complete it by Dirichlet boundary conditions on $\partial \omega$ : there exists a unique solution $\zeta_{*}^{2 \ell}$ to the problem

$$
\left\{\begin{aligned}
& \zeta_{*} \in H^{1}(\omega)^{2} \quad \text { and }\left.\quad \zeta_{*}\right|_{\partial \omega}=h_{*}^{2 \ell}, \\
& \forall v_{*} \in \stackrel{\circ}{H^{1}(\omega)^{2},} \quad \int_{\omega} M_{\alpha \beta \gamma \delta} e_{\gamma \delta}\left(\zeta_{*}\right) e_{\alpha \beta}\left(v_{*}\right)=-\frac{1}{2} \int_{\omega} F_{\alpha}\left(v^{2 \ell}\right) v_{\alpha} .
\end{aligned}\right.
$$

For this choice of $\zeta_{*}^{2 \ell}$, the compatibility condition (2.16a) holds for $k=\ell+1$. As the matrix $\left(A_{\alpha 3 \gamma 3}\right)$ is invertible (see $\left.(2.8)\right)$ problem $(2.16 \mathrm{~b})-(2.16 \mathrm{c})$ is solvable.

In order to investigate the compatibility condition $(2.15 \mathrm{a})$ for $k=\ell+2$, we split $v_{3}^{2 \ell+2}$ into two pieces

$$
\begin{aligned}
v_{3}^{2 \ell+2}= & y_{3}^{2 \ell+2}+z_{3}^{2 \ell+2} \text { s.t. } \int_{-1}^{+1} y_{3}^{2 \ell+2} d x_{3}=\int_{-1}^{+1} z_{3}^{2 \ell+2} d x_{3}=0 \\
\text { and } \quad & y_{3}^{2 \ell+2} \text { sol. of }(2.15) \text { with } u^{2 \ell}=v^{2 \ell}+\left(\zeta_{*}^{2 \ell}, 0\right) \\
& z_{3}^{2 \ell+2} \text { sol. of }(2.15) \text { with } u^{2 \ell}=\left(-x_{3} \nabla_{*} \zeta_{3}^{2 \ell}, \zeta_{3}^{2 \ell}\right)
\end{aligned}
$$

$y_{3}^{2 \ell+2}$ is now completely determined whereas $z_{3}^{2 \ell+2}$ has yet to be constructed.

Correspondingly, we split $v_{*}^{2 \ell+2}$ into two pieces

$$
\begin{aligned}
& v_{*}^{2 \ell+2}=y_{*}^{2 \ell+2}+z_{*}^{2 \ell+2} \quad \text { s.t. } \int_{-1}^{+1} y_{*}^{2 \ell+2} d x_{3}=\int_{-1}^{+1} z_{*}^{2 \ell+2} d x_{3}=0 \\
& \text { and } \quad y_{*}^{2 \ell+2} \text { sol. of (2.16) with } u^{2 \ell}=v^{2 \ell}+\left(\zeta_{*}^{2 \ell}, 0\right) \text { and } u_{3}^{2 \ell+2}=y_{3}^{2 \ell+2} \\
& z_{*}^{2 \ell+2} \text { sol. of }(2.16) \text { with } u^{2 \ell}=\left(-x_{3} \nabla_{*} \zeta_{3}^{2 \ell}, \zeta_{3}^{2 \ell}\right) \text { and } u_{3}^{2 \ell+2}=z_{3}^{2 \ell+2} \text {. }
\end{aligned}
$$

Then condition (2.15a) for $k=\ell+2$ reduces to

$$
\partial_{\alpha} A_{\alpha 3 \gamma 3} \int_{-1}^{+1} e_{\gamma 3}\left(z^{2 \ell+2}\right) d x_{3}=-\partial_{\alpha} A_{\alpha 3 \gamma 3} \int_{-1}^{+1} e_{\gamma 3}\left(y^{2 \ell+2}\right) d x_{3} .
$$

The relation (2.16b) for $k=\ell+1$ gives us, by definition of $z^{2 \ell+2}$ :

$$
2 A_{\alpha 3 \gamma 3} \partial_{3} e_{\gamma 3}\left(z^{2 \ell+2}\right)=-\partial_{\beta} A_{\alpha \beta 33} \partial_{3} z_{3}^{2 \ell+2}-\partial_{\beta} A_{\alpha \beta \gamma \delta} e_{\gamma \delta}\left(-x_{3} \nabla_{*} \zeta_{3}^{2 \ell}\right) .
$$

But we easily compute that

$$
z_{3}^{2 \ell+2}=A_{3333}^{-1} A_{33 \gamma \delta}\left(\frac{x_{3}^{2}}{2}-\frac{1}{6}\right) \partial_{\gamma \delta} \zeta_{3}^{2 \ell} .
$$

Whence the relation

$$
2 A_{\alpha 3 \gamma 3} \partial_{3} e_{\gamma 3}\left(z^{2 \ell+2}\right)=-x_{3} \partial_{\beta} A_{\alpha \beta 33} A_{3333}^{-1} A_{33 \gamma \delta} \partial_{\gamma \delta} \zeta_{3}^{2 \ell}+x_{3} \partial_{\beta} A_{\alpha \beta \gamma \delta} \partial_{\gamma \delta} \zeta_{3}^{2 \ell} .
$$

Integrating from -1 to $x_{3}$ and taking advantage of the relation (2.16c) for $k=\ell+1$ with $u^{2 \ell+2}=z^{2 \ell+2}$ and $u^{2 \ell}=\left(-x_{3} \nabla_{*} \zeta_{3}^{2 \ell}, \zeta_{3}^{2 \ell}\right)$ we obtain

$$
2 A_{\alpha 3 \gamma 3} e_{\gamma 3}\left(z^{2 \ell+2}\right)=\left(\frac{x_{3}^{2}}{2}-\frac{1}{2}\right)\left(-\partial_{\beta} A_{\alpha \beta 33} A_{3333}^{-1} A_{33 \gamma \delta} \partial_{\gamma \delta} \zeta_{3}^{2 \ell}+\partial_{\beta} A_{\alpha \beta \gamma \delta} \partial_{\gamma \delta} \zeta_{3}^{2 \ell}\right) \text {. }
$$


Integrating once more in $x_{3}$ from -1 to +1 and derivating with respect to $x_{\alpha}$, we reduce condition $(2.20)$ to

$$
-\partial_{\alpha \beta} A_{\alpha \beta 33} A_{3333}^{-1} A_{33 \gamma \delta} \partial_{\gamma \delta} \zeta_{3}^{2 \ell}+\partial_{\alpha \beta} A_{\alpha \beta \gamma \delta} \partial_{\gamma \delta} \zeta_{3}^{2 \ell}=\frac{3}{2} F_{3}\left(y^{2 \ell+2}\right)
$$

where

$$
F_{3}\left(y^{2 \ell+2}\right)=2 \partial_{\alpha} A_{\alpha 3 \gamma 3} \int_{-1}^{+1} e_{\gamma 3}\left(y^{2 \ell+2}\right) d x_{3} .
$$

Thus (2.21) is the equation of bending in $\omega$ associated with the rigidity matrix $M_{\alpha \beta \gamma \delta}$ defined in (2.18). We can complete it by Dirichlet boundary conditions on $\partial \omega$ : there exists a unique solution $\zeta_{3}^{2 \ell}$ to the problem

$$
\left\{\begin{aligned}
\zeta_{3} & \in H^{2}(\omega) \quad \text { and }\left.\quad \zeta_{3}\right|_{\partial \omega}=h_{3}^{2 \ell},\left.\quad \partial_{r} \zeta_{3}\right|_{\partial \omega}=h_{n}^{2 \ell}, \\
\forall v_{3} & \in \stackrel{\circ}{H^{2}}(\omega), \quad \int_{\omega} M_{\alpha \beta \gamma \delta} \partial_{\gamma \delta}\left(\zeta_{3}\right) \partial_{\alpha \beta}\left(v_{3}\right)=\frac{3}{2} \int_{\omega} F_{3}\left(y^{2 \ell+2}\right) v_{3} .
\end{aligned}\right.
$$

Finally, the Kirchhoff-Love displacement $u_{\mathrm{KL}}^{2 \ell}$ is completely determined and the compatibility conditions (2.16a), resp. (2.15a), hold for $k=\ell+1$, resp. $k=\ell+2$, which ensures the existence and the uniqueness of $v^{2 \ell+2}$ satisfying the zero mean condition over $x_{3}$. The induction condition $\left(\mathscr{I}^{\ell+1}\right)$ is proved.

\subsection{An even Ansatz for problem (1.6)}

The above algorithm can also be applied to the situation of problem (1.6), with the right-hand side $f(\varepsilon)=f^{0}$. In this case, we set $f^{k}=0$ for any non zero integer $k$. But Theorem 2.4 below applies in the case when $f(\varepsilon)$ has an expansion like (1.5) with only even order terms. Then, instead of problems (2.4) and (2.5) we obtain

$$
\begin{aligned}
& u_{3}^{2 k}=0 \text { on } \Gamma_{0} \\
& A_{3333} \partial_{33} u_{3}^{2 k}+A_{33 \gamma \delta} \partial_{3} e_{\gamma \delta}\left(u^{2 k-2}\right)+2 \partial_{\alpha} A_{\alpha 3 \gamma 3} e_{\gamma 3}\left(u^{2 k-2}\right)=-f_{3}^{2 k-4} \text { in } \Omega, \\
& A_{3333} \partial_{3} u_{3}^{2 k}+A_{33 \gamma \delta} e_{\gamma \delta}\left(u^{2 k-2}\right)=0 \text { on }\left\{x_{3}= \pm 1\right\} \cap \partial \Omega, \\
& u_{\alpha}^{2 k}=0 \text { on } \Gamma_{0}, \\
& 2 A_{\alpha 3 \gamma 3} \partial_{3} e_{\gamma 3}\left(u^{2 k}\right)+\partial_{\beta} A_{\alpha \beta 33} \partial_{3} u_{3}^{2 k}+\partial_{\beta} A_{\alpha \beta \gamma \delta} e_{\gamma \delta}\left(u^{2 k-2}\right)=-f_{\alpha}^{2 k-2} \text { in } \Omega, \\
& A_{\alpha 3 \gamma 3} e_{\gamma 3}\left(u^{2 k}\right)=0 \text { on }\left\{x_{3}= \pm 1\right\} \cap \partial \Omega
\end{aligned}
$$

Theorem 2.4 Let for any $k \in \mathbb{N}$, a function $f^{2 k}$ be given in $\mathscr{C}^{\infty}(\bar{\Omega})^{3}$. Moreover, we set $u^{-2}=0$. Then, for any $k \in \mathbb{N}$, there exist a unique displacement $v^{2 k}$ and $a$ unique Kirchhoff-Love displacement $u_{\mathrm{KL}}^{2 k}$ such that

$$
\left\{\begin{array}{l}
u^{2 k}:=v^{2 k}+u_{\mathrm{KL}}^{2 k} \text { is solution of }(2.23 \mathrm{~b})-(2.23 \mathrm{c}) \&(2.24 \mathrm{~b})-(2.24 \mathrm{c}), \\
\forall x_{*} \in \bar{\omega}, \quad \int_{-1}^{+1} v^{2 k}\left(x_{*}, x_{3}\right) d x_{3}=0, \\
u_{\mathrm{KL}}^{2 k}=\left(\zeta_{*}^{2 k}-x_{3} \nabla_{*} \zeta_{3}^{2 k}, \zeta_{3}^{2 k}\right) \quad \text { and }\left.\quad \zeta_{3}^{2 k}\right|_{\partial \omega}=0,\left.\quad \partial_{r} \zeta_{3}^{2 k}\right|_{\partial \omega}=0,\left.\quad \zeta_{*}^{2 k}\right|_{\partial \omega}=0 .
\end{array}\right.
$$


The functions $v^{2 k}$ and $u_{\mathrm{KL}}^{2 k}$ are smooth on $\bar{\Omega}$.

The proof is similar to that of Theorem 2.3 and our calculations imply that the generating functions $\zeta_{*}^{0}$ and $\zeta_{3}^{0}$ of $u_{\mathrm{KL}}^{0}$ are respectively the solutions of the membrane equations

$$
\zeta_{*}^{0} \in \stackrel{\circ}{H}^{1}(\omega)^{2} \quad \text { and } \quad-\partial_{\beta} M_{\alpha \beta \gamma \delta} e_{\gamma \delta}\left(\zeta_{*}^{0}\right)=\frac{1}{2} \int_{-1}^{+1} f_{\alpha}^{0} d x_{3}
$$

and the bending equation

$$
\zeta_{3}^{0} \in \stackrel{\circ}{H}^{2}(\omega) \quad \text { and } \quad \partial_{\alpha \beta} M_{\alpha \beta \gamma \delta} \partial_{\gamma \delta}\left(\zeta_{3}^{0}\right)=\frac{3}{2} \int_{-1}^{+1}\left(f_{3}^{0}+x_{3} \partial_{\alpha} f_{\alpha}^{0}\right) d x_{3} .
$$

Despite the fact that we may impose zero Dirichlet boundary conditions on the

Kirchhoff-Love parts $u_{\mathrm{KL}}^{2 k}$ of the Ansatz, it is impossible to prescribe the Dirichlet traces of the parts $v^{2 k}$ with zero mean values. Since $v^{0}=0$, the traces of $u^{0}(x)+$ $\varepsilon^{2} u^{2}(x)+\cdots$ on $\Gamma_{0}$ behave like $\mathcal{O}\left(\varepsilon^{2}\right)$ but they are not zero in general. These traces indicate the existence of boundary layers in the neighborhood of $\Gamma_{0}$.

\section{THE REDUCED-NORMAL PROBLEM ON A SEMI-INFINITE STRIP FOR THE DETERMINATION OF THE BOUNDARY LAYER ANSATZ}

\subsection{Guess of the boundary layers}

As we have just seen, the even Ansatz formally associated with $u(\varepsilon)$, namely

$$
u^{+}(\varepsilon):=u^{0}(x)+\varepsilon^{2} u^{2}(x)+\cdots+\varepsilon^{2 k} u^{2 k}(x)+\cdots
$$

yields a sort of approximation of $u(\varepsilon)$ which, in the sense of asymptotic expansions, satisfies

$$
\begin{aligned}
u^{+}(\varepsilon) & \in H^{1}(\Omega)^{3}, \\
\forall v & \in V(\Omega), \quad \int_{\Omega} A \kappa(\varepsilon)\left(u^{+}(\varepsilon)\right): \kappa(\varepsilon)(v)=\int_{\Omega} f \cdot v,
\end{aligned}
$$

but $u^{+}(\varepsilon)$ does not belong to $V(\Omega)$. Only the leading Kirchhoff-Love term $u^{0}$ belongs to $V(\Omega)$ and, indeed, it coincides with the limit of $u(\varepsilon)$ for the topology of $H^{1}(\Omega)^{3}$ as $\varepsilon \rightarrow 0, c f$. [8], [14], [15], [9] and [7].

Since $u(\varepsilon)-u^{0}$ belongs to $V(\Omega)$, we may hope that the even Ansatz can be completed by a boundary layer series so that we obtain the true complete asymptotics of $u(\varepsilon)$. By boundary layer term we mean a function defined on $\bar{\Omega}$ with its support concentrated in the neighborhood of $\Gamma_{0}$, the "width" of its support behaving like $\varepsilon$.

Such a sort of function would be conveniently described in the local coordinates $\left(r, s, x_{3}\right)$ where $r$ denotes the variable counted along the reentrant unit normal $n_{r}$ 
to $\partial \omega$ and $s \in S$ is a curvilinear coordinate along $\partial \omega$, see (2.10)-(2.11). Then a boundary layer term is given by a function of the type

$$
w(\varepsilon):\left(r, s, x_{3}\right) \longmapsto w\left(\frac{r}{\varepsilon}, s, x_{3}\right)
$$

where $w$ is a function of $\left(t, s, x_{3}\right) \in \mathbb{R}^{+} \times S \times(-1,+1)$ and is called profile. We intend to search profiles $w$ rapidly decreasing with respect to $t$ which could compensate for the Dirichlet traces of $u^{+}(\varepsilon)-u^{0}=\varepsilon^{2} u^{2}(x)+\cdots+$. Ideally, $w(\varepsilon)$ has to fulfill the same Dirichlet conditions as $\varepsilon^{2} u^{2}(x)+\cdots+$, the same Neumann conditions on $\left\{x_{3}= \pm 1\right\} \cap \partial \Omega$ and also the homogeneous interior elasticity system inside $\Omega$.

In this $\S 3$, we construct the equations governing the boundary layer terms and exhibit the behavior as $t \rightarrow+\infty$ of the solutions of these equations. In $\S 4$, we will adjust the different terms to obtain the complete asymptotics of $u$. As already hinted in the introduction, we will see that this is more complicated than the mere juxtaposition of the even Ansatz and a boundary layer series.

\subsection{Position of the reduced-normal problem}

We are interested in solving a problem with non-homogeneous Dirichlet traces like $(2.1)$, in the new coordinates $\left(r, s, x_{3}\right)$. The change of variables $x_{*}=\left(x_{1}, x_{2}\right) \mapsto$ $(r, s)$, with $x_{*}=X_{*}(r, s)$ and the corresponding change in the displacements $u_{*} \mapsto$ $\left(u_{r}, u_{s}\right)$ is such that, $c f(2.11)-(2.14)$ :

$$
\left(\begin{array}{c}
\partial_{1} \\
\partial_{2}
\end{array}\right)=D X_{*}\left(\begin{array}{c}
\partial_{r} \\
\partial_{s}
\end{array}\right) \text { and }\left(\begin{array}{l}
u_{1} \\
u_{2}
\end{array}\right)=D X_{*}\left(\begin{array}{c}
u_{r} \\
u_{s}
\end{array}\right) .
$$

As a consequence, if we define the new scaled tensor $\tilde{\kappa}(\varepsilon)$ similarly to $\kappa(\varepsilon)$ in $(1.4 \mathrm{a})$ :

$$
\begin{aligned}
\tilde{\kappa}_{\alpha \beta}(\varepsilon)(v)=e_{\alpha \beta}\left(\partial_{r}, \partial_{s}, \partial_{3}\right)(v), \\
\tilde{\kappa}_{\alpha 3}(\varepsilon)(v)=\varepsilon^{-1} e_{\alpha 3}\left(\partial_{r}, \partial_{s}, \partial_{3}\right)(v), \\
\tilde{\kappa}_{33}(\varepsilon)(v)=\varepsilon^{-2} e_{33}\left(\partial_{r}, \partial_{s}, \partial_{3}\right)(v),
\end{aligned}
$$

where $e_{11}\left(\partial_{r}, \partial_{s}, \partial_{3}\right)(v)=\partial_{r} u_{r}, \quad e_{12}\left(\partial_{r}, \partial_{s}, \partial_{3}\right)(v)=\frac{1}{2}\left(\partial_{r} u_{s}+\partial_{s} u_{r}\right)$, etc... Problem (2.1) is transformed into a similar problem in the domain $\widetilde{\Omega}=(0, \rho) \times S \times(-1,+1)$ with "lateral face" $\widetilde{\Gamma}_{0}=\{0\} \times S \times(-1,+1)$

$$
\begin{aligned}
& w(\varepsilon) \in H^{1}(\widetilde{\Omega})^{3} \quad \text { and } \quad w(\varepsilon)=h(\varepsilon) \quad \text { on } \quad \widetilde{\Gamma}_{0}, \\
& \forall v \in V(\widetilde{\Omega}), \quad \int_{\widetilde{\Omega}} \tilde{A} \tilde{\kappa}(\varepsilon)(w(\varepsilon)): \tilde{\kappa}(\varepsilon)(v)+\widetilde{\mathcal{A}}^{\prime}(w(\varepsilon), v)=0,
\end{aligned}
$$

where the $9 \times 9$ matrix $\widetilde{A}=\widetilde{A}(r, s)$ has the same features as $A$ (positivity, smoothness and property (1.3) on the coefficients) and $\widetilde{\mathcal{A}}^{\prime}$ is a first order integro-differential form; here $\boldsymbol{i}$ and $\boldsymbol{j}$ denote multi-indices in $\mathbb{N}^{3}$ :

$$
\widetilde{\mathcal{A}}^{\prime}(w, v)=\sum_{|\boldsymbol{i}|+|\boldsymbol{j}| \leq 1} \int_{\widetilde{\Omega}} c_{\boldsymbol{i j}}(r, s) \partial^{i} w \partial^{j} v
$$


Keeping in mind that we want solutions of the form (3.3), we apply to problem (3.4) the change of variables

$$
\left(r, s, x_{3}\right) \longmapsto\left(t, s, x_{3}\right) \quad \text { where } \quad t=\frac{r}{\varepsilon}
$$

thus $\partial_{t}=\varepsilon \partial_{r}$ and for purposes of homogeneity we introduce the following change in the displacements

$$
\left(w_{r}, w_{s}, w_{3}\right) \longmapsto\left(\varphi_{t}, \varphi_{s}, \varphi_{3}\right)=\left(\varepsilon w_{r}, \varepsilon w_{s}, w_{3}\right) .
$$

Now, problem (3.4) is transformed into the problem on $\widetilde{\Sigma}:=\mathbb{R}^{+} \times S \times(-1,+1)$ :

$$
\begin{aligned}
& \varphi \in H^{1}(\widetilde{\Sigma})^{3} \quad \text { and } \quad \varphi=g \quad \text { on } \quad \widetilde{\Gamma}_{0}, \\
& \forall v \in V(\widetilde{\Sigma}), \quad \int_{\widetilde{\Sigma}} \widetilde{A}(\varepsilon t, s) e\left(\partial_{t}, 0, \partial_{3}\right)(\varphi): e\left(\partial_{t}, 0, \partial_{3}\right)(v)+\sum_{k=1}^{4} \varepsilon^{k} \widetilde{\mathcal{A}}^{k}(\varphi, v)=0,
\end{aligned}
$$

where $\widetilde{\mathcal{A}}^{k}$ is a second order integro-differential form:

$$
\widetilde{\mathcal{A}}^{k}(\varphi, v)=\sum_{|\boldsymbol{i}|,|\boldsymbol{j}| \leq 1} \int_{\widetilde{\Sigma}} c_{i j}^{k}(\varepsilon t, s) \partial^{i} \varphi \partial^{j} v .
$$

We have also to take into account the variable $\varepsilon t$ in the coefficients of the operators. With the help of the Taylor expansion of the coefficients $c_{\boldsymbol{i} j}^{k}$ in $t=0$, we obtain for problem (3.6) the formal expansion

$$
\begin{aligned}
& \varphi \in H^{1}(\widetilde{\Sigma})^{3} \quad \text { and } \quad \varphi=g \quad \text { on } \quad \widetilde{\Gamma}_{0}, \\
& \forall v \in V(\widetilde{\Sigma}), \quad \int_{\widetilde{\Sigma}} \widetilde{A}(0, s) e\left(\partial_{t}, 0, \partial_{3}\right)(\varphi): e\left(\partial_{t}, 0, \partial_{3}\right)(v)+\sum_{k \geq 1} \varepsilon^{k} \mathcal{B}^{k}(\varphi, v)=0,
\end{aligned}
$$

where for any $k \geq 1, \mathcal{B}^{k}$ is a second order integro-differential form:

$$
\mathcal{B}^{k}(\varphi, v)=\sum_{|\boldsymbol{i}|,|\boldsymbol{j}| \leq 1} \int_{\widetilde{\Sigma}} b_{i \boldsymbol{j}}^{k}(t, s) \partial^{i} \varphi \partial^{j} v
$$

whose coefficients $b_{i j}^{k}$ are polynomials of $t$ for each fixed $s$. Of course, we obtain finite expansions with remainders by Taylor formulas.

Thus, we see that the "principal part" (in the sense of the powers of $\varepsilon$ ) of this problem does not involve any more the tangential derivation $\partial_{s}$ : thus the variable $s$ is a mere parameter.

Definition 3.1 The matrix $\widetilde{A}(r, s)$ being the matrix transformed from $A$ by the change of variables $\left(x_{1}, x_{2}\right) \mapsto(r, s)$, we denote by $B$ the matrix

$$
B=B(s)=\left(B_{i j k l}\right)=\widetilde{A}(0, s) .
$$

The matrix $B$ is positive definite, depends smoothly on $s \in S$ and its coefficients satisfy the property (1.3). Let $\Sigma^{+}$and $\gamma_{0}$ denote the semi-infinite strip and its lateral face:

$$
\Sigma^{+}=\left\{\left(t, x_{3}\right) \mid t>0,-1<x_{3}<+1\right\} \quad \text { and } \quad \gamma_{0}=\left\{\left(t, x_{3}\right) \mid t=0,-1<x_{3}<+1\right\} .
$$


The space of admissible displacements on $\Sigma^{+}$is

$$
V\left(\Sigma^{+}\right)=\left\{v \in H^{1}\left(\Sigma^{+}\right)^{3} \mid \quad v=0 \text { on } \gamma_{0}\right\}
$$

For each $s$ fixed in $S$, the reduced-normal problem is the mixed Dirichlet-Neumann problem in $\Sigma^{+}$:

$$
\begin{aligned}
& \varphi \in H^{1}\left(\Sigma^{+}\right)^{3} \quad \text { and }\left.\quad \varphi\right|_{\gamma_{0}}=g \\
& \forall v \in V\left(\Sigma^{+}\right), \quad \int_{\Sigma^{+}} B(s) e\left(\partial_{t}, 0, \partial_{3}\right)(\varphi): e\left(\partial_{t}, 0, \partial_{3}\right)(v)=0,
\end{aligned}
$$

with unknown $\varphi=\left(\varphi_{t}, \varphi_{s}, \varphi_{3}\right)$ and data $g=\left(g_{t}, g_{s}, g_{3}\right)$.

\subsection{Behavior at infinity of solutions of the reduced-normal problem}

The above problem (3.9) is an elasticity problem associated with the linearized strain tensor $e\left(\partial_{t}, 0, \partial_{3}\right)$. So we can expect that the space $\mathcal{R}$ of the rigid displacements associated with the tensor $e\left(\partial_{t}, 0, \partial_{3}\right)$ will play an important role:

$$
R \in \mathcal{R} \quad \Longleftrightarrow \quad e\left(\partial_{t}, 0, \partial_{3}\right)(R)=0
$$

This means explicitly:

$$
\partial_{t} R_{t}=0, \quad \partial_{3} R_{3}=0 ; \quad \partial_{t} R_{s}=0, \quad \partial_{3} R_{s}=0 ; \quad \text { and } \quad \partial_{t} R_{3}+\partial_{3} R_{t}=0 .
$$

This implies that the pair $\left(R_{t}, R_{3}\right)$, as a function of the variables $\left(t, x_{3}\right)$, is a rigid displacement whereas $R_{s}$ is constant. So we find that

$$
\mathcal{R}=\left\{R=\left(R_{t}, R_{s}, R_{3}\right)=\left(c_{t}, c_{s}, c_{3}\right)+c_{n}\left(-x_{3}, 0, t\right) \mid c_{t}, c_{s}, c_{3}, c_{n} \in \mathbb{R}\right\} .
$$

The following result states that the solutions of problem (3.9) are exponentially decreasing towards rigid displacements as the distance to the clamped face $\gamma_{0}$ becomes "large".

Theorem 3.2 Let $s$ belong to $S$. For all $g \in H^{1 / 2}\left(\gamma_{0}\right)^{3}$ there exists a unique rigid displacement $R=R(s)(g) \in \mathcal{R}$ so that the problem

$$
\begin{aligned}
& \varphi \in H^{1}\left(\Sigma^{+}\right)^{3} \quad \text { and }\left.\quad \varphi\right|_{\gamma_{0}}=g+\left.R\right|_{\gamma_{0}}, \\
& \forall v \in V\left(\Sigma^{+}\right), \quad \int_{\Sigma^{+}} B(s) e\left(\partial_{t}, 0, \partial_{3}\right)(\varphi): e\left(\partial_{t}, 0, \partial_{3}\right)(v)=0,
\end{aligned}
$$

has a (unique) solution. Moreover, there is $\eta_{0}>0$, which does not depend on $g$, such that $e^{\eta_{0} t} \varphi\left(t, x_{3}\right)$ is bounded on $\Sigma^{+}$.

Indeed, in order to take account of nonzero right hand-sides coming from the operators $\mathcal{B}^{k}$ of formula (3.7b), we are led to use the following more general statement. The natural spaces involved require an exponential decay at infinity: 
Definition 3.3 For $\eta \in \mathbb{R}$ let $H_{\eta}^{1}\left(\Sigma^{+}\right)$be the space of functions $v$ such that $e^{\eta t} v$ belongs to $H^{1}\left(\Sigma^{+}\right)$and let $V_{\eta}^{\prime}\left(\Sigma^{+}\right)$be the space of distributions $w=\left(w_{t}, w_{s}, w_{3}\right)$ such that $e^{\eta t} w$ belongs to the dual space of $V\left(\Sigma^{+}\right)$. Let $\boldsymbol{K}_{\eta}^{1}\left(\Sigma^{+}\right)$and $\boldsymbol{K}_{\eta}^{-1}\left(\Sigma^{+}\right)$ be the corresponding intersection spaces

$$
\boldsymbol{K}_{\eta}^{1}\left(\Sigma^{+}\right)=\bigcap_{\bar{\eta}<\eta} H_{\bar{\eta}}^{1}\left(\Sigma^{+}\right)^{3} \quad \text { and } \quad \boldsymbol{K}_{\eta}^{-1}\left(\Sigma^{+}\right)=\bigcap_{\bar{\eta}<\eta} V_{\bar{\eta}}^{\prime}\left(\Sigma^{+}\right) .
$$

We define $\boldsymbol{K}_{\eta}^{ \pm 1}\left(\Omega \times \mathbb{R}^{+}\right)$in a similar way (such spaces describe the regularity of complete profiles $\Psi(x, t))$.

Corollary 3.4 For all $g \in H^{1 / 2}\left(\gamma_{0}\right)^{3}$ and for all $f \in \boldsymbol{K}_{\eta_{0}}^{-1}\left(\Sigma^{+}\right)$there exists a unique rigid displacement $R=R(s)(g, f) \in \mathcal{R}$ so that the problem

$$
\begin{aligned}
& \varphi \in H^{1}\left(\Sigma^{+}\right)^{3} \quad \text { and }\left.\quad \varphi\right|_{\gamma_{0}}=g+\left.R\right|_{\gamma_{0}}, \\
& \forall v \in V\left(\Sigma^{+}\right), \quad \int_{\Sigma^{+}} B e\left(\partial_{t}, 0, \partial_{3}\right)(\varphi): e\left(\partial_{t}, 0, \partial_{3}\right)(v)=\int_{\Sigma^{+}} f v,
\end{aligned}
$$

has a (unique) solution. Moreover, this solution $\varphi$ belongs to $\boldsymbol{K}_{\eta_{0}}^{1}\left(\Sigma^{+}\right)$.

\section{COMPLETE ASYMPTOTICS}

\subsection{The first terms in the boundary layer series}

We consider once more "the" even Ansatz (3.1) that we have constructed in $\S 2$. Let us recall that each component $u^{2 k}$ is the sum of a term $v^{2 k}$ with null means with respect to $x_{3}$ and of a Kirchhoff-Love displacement $u_{\mathrm{KL}}^{2 k}$. Only two components are completely determined: viz. the first Kirchhoff-Love displacement $u_{\mathrm{KL}}^{0}$ whose generating functions $\zeta_{*}^{0}$ and $\zeta_{3}^{0}$ are the unique solutions of problems (2.25) and (2.26) respectively, and the component $v^{2}$ which only depends on $u^{0}$.

The leading term in the Dirichlet trace of the even Ansatz $u^{+}(\varepsilon)$ is $\left.\varepsilon^{2} u^{2}\right|_{\Gamma_{0}}$. We propose as first term of the boundary layer series

$$
\left(\varepsilon w_{r}^{1}, \varepsilon w_{s}^{1}, \varepsilon^{2} w_{3}^{2}\right)\left(\frac{r}{\varepsilon}, s, x_{3}\right)
$$

which, by the change of functions (3.5), corresponds to the new unknown

$$
\varepsilon^{2} \varphi=\left(\varepsilon^{2} w_{r}^{1}, \varepsilon^{2} w_{s}^{1}, \varepsilon^{2} w_{3}^{2}\right)
$$

This is the reason for this curious inhomogeneous form. This "first term" compensates only for the trace of $\varepsilon^{2} u_{3}^{2}$. So, for each fixed $s \in S$ we search for a solution $\varphi(s)\left(t, x_{3}\right)$ of problem $(c f .(3.9))$

$$
\left\{\begin{aligned}
\varphi(s) & \in H^{1}\left(\Sigma^{+}\right)^{3} \quad \text { and }\left.\quad \varphi(s)\right|_{\gamma_{0}}=g(s), \\
\forall v & \in V\left(\Sigma^{+}\right), \quad \int_{\Sigma^{+}} B(s) e\left(\partial_{t}, 0, \partial_{3}\right)(\varphi(s)): e\left(\partial_{t}, 0, \partial_{3}\right)(v)=0
\end{aligned}\right.
$$


with data $g(s)=\left(0,0,\left.u_{3}^{2}(s)\right|_{\gamma_{0}}\right)$. According to Theorem 3.2, we know that, for each fixed $s \in S$, there exists a rigid displacement $R=R(s) \in \mathcal{R}$ so that problem (4.2) with the new data $g(s)=\left(0,0,\left.u_{3}^{2}(s)\right|_{\gamma_{0}}\right)+\left.R(s)\right|_{\gamma_{0}}$ has an exponentially decreasing solution, that we denote $\varphi^{2}(s)$. Then the Dirichlet traces of $\varphi^{2}$ are

$$
\left.\varphi_{t}^{2}(s)\right|_{\gamma_{0}}=c_{t}(s)-c_{n}(s) x_{3},\left.\quad \varphi_{s}^{2}(s)\right|_{\gamma_{0}}=c_{s}(s),\left.\quad \varphi_{3}^{2}(s)\right|_{\gamma_{0}}=c_{3}(s)+\left.u_{3}^{2}(s)\right|_{\gamma_{0}}
$$

and returning to the boundary layer Ansatz (4.1):

$$
\left.\varepsilon w_{r}^{1}\right|_{\gamma_{0}}=\varepsilon\left(c_{t}-c_{n} x_{3}\right),\left.\quad \varepsilon w_{s}^{1}\right|_{\gamma_{0}}=\varepsilon c_{s},\left.\quad \varepsilon^{2} w_{3}^{2}\right|_{\gamma_{0}}=\varepsilon^{2}\left(c_{3}+\left.u_{3}^{2}\right|_{\gamma_{0}}\right) .
$$

The additional trace $c_{3}$ in the last term causes no problem because we know that $u^{2}=v^{2}+u_{\mathrm{KL}}^{2}$ with $v^{2}$ uniquely determined, but the traces of the generating functions $\zeta_{*}^{2}$ and $\zeta_{3}^{2}$ remain to be chosen. In particular we can impose that $\left.\zeta_{3}^{2}\right|_{\partial \omega}=c_{3}$.

The occurrence of the traces $c_{t}, c_{n}$ and $c_{s}$ at the level of $\varepsilon$ is somewhat unexpected. Indeed, we may compensate for them by the help of a new Kirchhoff-Love displacement $u_{\mathrm{KL}}^{1}$ which should satisfy the following properties: its traces $u_{\mathrm{KL}, r}^{1}$ and $u_{\mathrm{KL}, s}^{1}$ have to coincide with $c_{t}-c_{n} x_{3}$ and $c_{s}$ respectively, whereas the trace of its third component $u_{\mathrm{KL}, 3}^{1}$ has to be 0 . Moreover, its generating functions $\zeta_{*}^{1}$ and $\zeta_{3}^{1}$ have to satisfy the equations inside $\omega$ governing the first term of an (odd) Ansatz for a problem similar to (2.1), cf. (2.19) and (2.22).

Using the formula $u_{\mathrm{KL}}^{1}=\left(\zeta_{*}^{1}-x_{3} \nabla_{*} \zeta_{3}^{1}, \zeta_{3}^{1}\right)$, we obtain that $\zeta_{*}^{1}$ and $\zeta_{3}^{1}$ are the unique solutions of

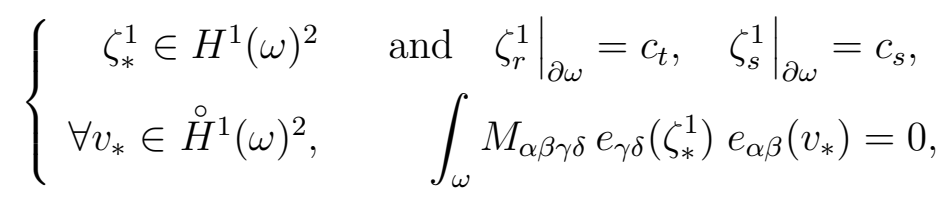

and

$$
\left\{\begin{aligned}
\zeta_{3}^{1} \in H^{2}(\omega) & \text { and }\left.\quad \zeta_{3}^{1}\right|_{\partial \omega}=0,\left.\quad \partial_{r} \zeta_{3}^{1}\right|_{\partial \omega}=c_{n}, \\
\forall v_{3} \in \stackrel{\circ}{H^{2}}(\omega), & \int_{\omega} M_{\alpha \beta \gamma \delta} \partial_{\gamma \delta}\left(\zeta_{3}^{1}\right) \partial_{\alpha \beta}\left(v_{3}\right)=0,
\end{aligned}\right.
$$

respectively (we recall that $M_{\alpha \beta \gamma \delta}=A_{\alpha \beta \gamma \delta}-A_{\alpha \beta 33} A_{3333}^{-1} A_{33 \gamma \delta}$ and that it is positive definite, see (2.18)).

Thus, in order to find one term of the boundary layer series, we have completely determined four traces, which corresponds exactly to the data at each step of the even Ansatz (Theorem 2.3).

\subsection{Asymptotic expansion of arbitrary order}

Here is our result concerning the formal solution of problem (1.6). 
Theorem 4.1 Let $f \in \mathscr{C}^{\infty}(\Omega)^{3}$. For each $k \in \mathbb{N}$ there exist a unique KirchhoffLove displacement $u_{\mathrm{KL}}^{k}$, a unique displacement $v^{k}$ such that $\int_{-1}^{1} v^{k}\left(x_{*}, x_{3}\right) d x_{3}=0$ for all $x_{*} \in \omega$, and a unique profile $w^{k} \in \boldsymbol{K}_{\eta_{0}}^{1}\left(\Sigma^{+}\right)$(see Corollary 3.4), such that the series

$$
U(\varepsilon)(x)=\sum_{k \in \mathbb{N}} \varepsilon^{k}\left(u_{\mathrm{KL}}^{k}(x)+v^{k}(x)-\chi(r) w^{k}\left(\frac{r}{\varepsilon}, s, x_{3}\right)\right)
$$

formally satisfies *

$$
\begin{aligned}
U(\varepsilon) & \in V(\Omega), \\
\forall v & \in V(\Omega), \quad \int_{\Omega} A \kappa(\varepsilon)(U(\varepsilon)): \kappa(\varepsilon)(v)=\int_{\Omega} f \cdot v .
\end{aligned}
$$

The smooth cut-off function $\chi$ is equal to 1 in a neighborhood of 0 and equal to 0 for $r \geq \rho$. The displacement $v^{0}$ is zero, and the profile $w^{0}$ is also zero. The third component $w_{3}^{1}$ of the profile $w^{1}$ is equal to 0 .

Proof. We make use of an induction argument. We reformulate problems (2.15) and (2.16) as:

$$
\begin{aligned}
& 2 \partial_{\alpha} A_{\alpha 3 \gamma 3} \int_{-1}^{+1} e_{\gamma 3}\left(u^{k-2}\right) d x_{3}=0 \\
& \left\{\begin{array}{l}
A_{3333} \partial_{33} u_{3}^{k}=-A_{33 \gamma \delta} \partial_{3} e_{\gamma \delta}\left(u^{k-2}\right)-2 \partial_{\alpha} A_{\alpha 3 \gamma 3} e_{\gamma 3}\left(u^{k-2}\right), \quad \text { in } \quad \Omega, \\
A_{3333} \partial_{3} u_{3}^{k}=-A_{33 \gamma \delta} e_{\gamma \delta}\left(u^{k-2}\right) \quad \text { on } \quad\left\{x_{3}= \pm 1\right\} \cap \partial \Omega,
\end{array}\right.
\end{aligned}
$$

and

$$
\begin{aligned}
& \partial_{\beta} A_{\alpha \beta 33} \int_{-1}^{+1} \partial_{3} u_{3}^{k} d x_{3}+\partial_{\beta} A_{\alpha \beta \gamma \delta} \int_{-1}^{+1} e_{\gamma \delta}\left(u^{k-2}\right) d x_{3}=0, \\
& \left\{\begin{array}{l}
A_{\alpha 3 \gamma 3} \partial_{33} u_{\gamma}^{k}=-A_{\alpha 3 \gamma 3} \partial_{3 \gamma} u_{3}^{k}-\partial_{\beta} A_{\alpha \beta 33} \partial_{3} u_{3}^{k}-\partial_{\beta} A_{\alpha \beta \gamma \delta} e_{\gamma \delta}\left(u^{k-2}\right) \text { in } \Omega, \\
A_{\alpha 3 \gamma 3} \partial_{3} u_{\gamma}^{k}=-A_{\alpha 3 \gamma 3} \partial_{\gamma} u_{3}^{k}, \quad \text { on } \quad\left\{x_{3}= \pm 1\right\} \cap \partial \Omega,
\end{array}\right.
\end{aligned}
$$

respectively.

Our induction hypothesis applies to the displacements $u_{\mathrm{KL}}^{k}$ and $v^{k}$, for $k \geq 0$, the traces $h_{r}^{k}, h_{n}^{k}, h_{s}^{k}$ and $h_{3}^{k}$ on $\partial \omega$, for $k \geq 0$, and the scaled profiles $\varphi^{k}$, for $k \geq 2$, which determine the profiles $w^{k}$ by

$$
\varphi_{t}^{k}=w_{t}^{k-1}, \quad \varphi_{s}^{k}=w_{s}^{k-1}, \quad \varphi_{3}^{k}=w_{3}^{k} .
$$

Let $\ell \geq 1$. Our induction hypothesis consists of the assertions (4.11)-(4.15) below and will be denoted $\left(\widetilde{\mathscr{I}}^{\ell}\right)$ in the sequel.

This means that for any $N \in \mathbb{N}$, if one cuts the series by $k \leq N$, defining $U^{N}$, then the trace $\left.U^{N}\right|_{\Gamma_{0}}$ is a $\mathcal{O}\left(\varepsilon^{N_{1}}\right)$ with $N_{1} \rightarrow \infty$ as $N \rightarrow \infty$, and the expansion with respect to $\varepsilon$ of $v \mapsto \int_{\Omega}(A \kappa(\varepsilon)(U(\varepsilon)): \kappa(\varepsilon)(v)-f \cdot v)$ in terms $\varepsilon^{k} \Phi^{k}(x, r / \varepsilon)$ with $\Phi^{k}(x, t) \in \boldsymbol{K}^{-1}\left(\Omega \times \mathbb{R}^{+}\right)$, yields a $\mathcal{O}\left(\varepsilon^{N_{2}}\right)$ with $N_{2} \rightarrow \infty$ as $N \rightarrow \infty$. This is different from the formal approach of [7] which, just like our algorithm for the "even Ansatz", does not include boundary layer terms. 
We suppose that the following are determined:

$$
\begin{array}{rlrl}
u_{\mathrm{KL}}^{k} & \text { for } k & =0, \ldots, 2 \ell-2, \\
v^{k} & \text { for } k & =0, \ldots, 2 \ell, \\
h_{r}^{k}, & h_{n}^{k}, h_{s}^{k} & \text { for } k=0, \ldots, 2 \ell-2, \\
h_{3}^{k} & \text { for } k=0, \ldots, 2 \ell-1, \\
\varphi^{k} & \text { for } k=1, \ldots, 2 \ell-1 .
\end{array}
$$

Letting $u^{k}=u_{\mathrm{KL}}^{k}+v^{k}$ and $u_{\mathrm{KL}}^{k}=\left(\zeta_{*}^{k}-x_{3} \nabla_{*} \zeta_{3}^{k}, \zeta_{3}^{k}\right)$, we have the following properties:

$$
\left.\zeta_{r}^{k}\right|_{\partial \omega}=h_{r}^{k},\left.\quad \zeta_{s}^{k}\right|_{\partial \omega}=h_{s}^{k},\left.\quad \zeta_{3}^{k}\right|_{\partial \omega}=h_{3}^{k},\left.\quad \partial_{r} \zeta_{3}^{k}\right|_{\partial \omega}=h_{n}^{k}
$$

for $k=0, \ldots, 2 \ell-2$, and

$$
\begin{array}{ll}
u^{k} \text { solution of }(4.8 \mathrm{~b})-(4.9 \mathrm{~b}) & \text { for } k=0, \ldots, 2 \ell-2, \\
v^{k} \text { solution of }(4.8 \mathrm{~b})-(4.9 \mathrm{~b}) & \text { for } k=2 \ell-1,2 \ell, \\
v^{k} \text { satisfies (4.8a) } & \text { for } k=2 \ell+1,2 \ell+2 .
\end{array}
$$

Moreover, for all $s \in S$, set:

$$
\begin{array}{r}
g_{t}^{k}=\left.v_{r}^{k-1}\right|_{\Gamma_{0}}+h_{r}^{k-1}-x_{3} h_{n}^{k-1}, \\
g_{s}^{k}=\left.v_{s}^{k-1}\right|_{\Gamma_{0}}+h_{s}^{k-1}-x_{3} \partial_{s} h_{3}^{k-1}, \\
g_{3}^{k}=\left.v_{3}^{k}\right|_{\Gamma_{0}}+h_{3}^{k},
\end{array}
$$

Then, $\varphi^{k}(s)$ belongs to $\boldsymbol{K}_{\eta_{0}}^{1}\left(\Sigma^{+}\right)$and is solution of (cf. problem (3.7)):

$$
\left\{\begin{array}{l}
\left.\varphi^{k}(s)\right|_{\gamma_{0}}=g^{k}(s) \\
\forall v \in V\left(\Sigma^{+}\right), \int_{\Sigma^{+}} B(s) e\left(\partial_{t}, 0, \partial_{3}\right)\left(\varphi^{k}\right): e\left(\partial_{t}, 0, \partial_{3}\right)(v)=-\int_{\Sigma^{+}} \sum_{j=1}^{k-1} \mathcal{B}^{j}\left(\varphi^{k-j}, v\right)
\end{array}\right.
$$

for $k=0, \ldots, 2 \ell-1$.

We initialize the recurrence by setting $u^{-2}=u^{-1}=0$, and we easily see that the condition $\left(\widetilde{\mathscr{I}}^{1}\right)$, i.e. (4.11)-(4.15) for $\ell=1$, holds with $u_{\mathrm{KL}}^{0}$ the first term of the even Ansatz, $v^{0}, v^{1}=0$ and $v^{2}$ the corresponding term in the even Ansatz, $h_{r}^{0}$, $h_{n}^{0}, h_{s}^{0}, h_{3}^{0}, h_{3}^{1}$ all equal to $0, \varphi^{1}=0$.

We assume that $\left(\widetilde{\mathscr{I}}^{\ell}\right)$ (namely (4.11)-(4.15)) holds for an $\ell \geq 1$ and we start proving $\left(\widetilde{\mathscr{I}}^{\ell+1}\right)$.

Our first step is the determination of $\varphi^{2 \ell}$. Since $\varphi^{k}$ belongs to $\boldsymbol{K}_{\eta_{0}}^{1}\left(\Sigma^{+}\right)$for $k=1, \ldots, 2 \ell-1$, then the right hand side of $(4.15), \sum_{j=1}^{2 \ell-1} \mathcal{B}^{j}\left(\varphi^{k-j}\right)$, belongs to $\boldsymbol{K}_{\eta_{0}}^{-1}\left(\Sigma^{+}\right)$. Then we can apply Corollary 3.4 and conclude that there exist $c_{r}(s)$, $c_{n}(s), c_{s}(s)$ and $c_{3}(s)$ such that problem (4.15) for $k=2 \ell$ and $g^{2 \ell}(s)$ defined by:

$$
\begin{array}{r}
g_{t}^{2 \ell}=\left.v_{r}^{2 \ell-1}\right|_{\Gamma_{0}}+c_{r}-x_{3} c_{n}, \\
g_{s}^{2 \ell}=\left.v_{s}^{2 \ell-1}\right|_{\Gamma_{0}}-x_{3} \partial_{s} h_{3}^{2 \ell-1}+c_{s}, \\
g_{3}^{2 \ell}=\left.v_{3}^{2 \ell}\right|_{\Gamma_{0}}+c_{3},
\end{array}
$$


admits a unique solution $\varphi^{2 \ell}$ belonging to $\boldsymbol{K}_{\eta_{0}}^{1}\left(\Sigma^{+}\right)$. Now we set:

$$
h_{r}^{2 \ell-1}=c_{r}, \quad h_{n}^{2 \ell-1}=c_{n}, \quad h_{s}^{2 \ell-1}=c_{s}, \quad h_{3}^{2 \ell}=c_{3} .
$$

Relying on the existence of the odd Ansatz including $u^{2 \ell-3}$ and $v^{2 \ell-1}$ and on the same argument used in the proof of the even Ansatz in Theorem 2.3, we deduce the existence of $u_{\mathrm{KL}}^{2 \ell-1}$ such that the boundary conditions (4.12) hold for $k=2 \ell-1$, and the existence of $v^{2 \ell+1}$, both of them satisfying ( $\left.c f .(4.13)\right)$ :

$$
\begin{array}{ll}
u^{k} \text { solution of }(4.8 \mathrm{~b})-(4.9 \mathrm{~b}) & \text { for } k=2 \ell-1, \\
v^{k} \text { solution of }(4.8 \mathrm{~b})-(4.9 \mathrm{~b}) & \text { for } k=2 \ell+1, \\
v^{2 \ell+1} \text { satisfies }(4.8 \mathrm{a}) & \text { for } k=2 \ell+3
\end{array}
$$

Next we determine $\varphi^{2 \ell+1}$ as before, which yields the values of $h_{r}^{2 \ell}, h_{n}^{2 \ell}$ and $h_{s}^{2 \ell}$. As $h_{3}^{2 \ell}$ was already determined in the previous step, we rely on the even Ansatz to deduce the existence of $u_{\mathrm{KL}}^{2 \ell}$ and $v^{2 \ell+2}$ fulfilling the conditions involved in $\left(\widetilde{\mathscr{I}}^{\ell+1}\right)$.

\subsection{Regularity of the terms of the polynomial Ansatz}

Let us assume that for a positive integer $m$, the right-hand side $f$ in problem (1.6) has the following regularity

$$
\left\{\begin{array}{l}
f_{\alpha} \in L^{2}\left(I, H^{m-1}(\omega)\right) \cap H^{m-1}\left(I, L^{2}(\omega)\right) \\
f_{3} \in H^{1}\left(I, H^{m-2}(\omega)\right) \cap H^{m-1}\left(I, L^{2}(\omega)\right) .
\end{array}\right.
$$

Here $I$ denotes the interval $(-1,+1)$. We note that, in fact $f_{\alpha}$ belongs to $H^{m-1}(\Omega)$ for $\alpha=1,2$ and that $f_{3}$ is a little less regular: $f_{3}$ and $\partial_{3} f_{3}$ both belong to $H^{m-2}(\Omega)$. Starting from (4.16), we are going to follow the algorithms of the proofs of Theorems 2.3 and 4.1 in order to determine the regularity of the different terms in the asymptotics of $u(\varepsilon)$.

As the midsurface $\omega$ is smooth, by the ellipticity of the membrane and bending problems, we obtain

$$
\zeta_{\alpha}^{0} \in H^{m+1}(\omega) \quad \text { and } \quad \zeta_{3}^{0} \in H^{m+2}(\omega)
$$

From equation (2.23b) we deduce that $\partial_{33} v_{3}^{2}$ belongs to $H^{\infty}\left(I, H^{m}(\omega)\right)$, whence solving a Neumann problem in the variable $x_{3}, v_{3}^{2}$ also belongs to $H^{\infty}\left(I, H^{m}(\omega)\right)$. Next, from equation $(2.24 \mathrm{~b})$ we deduce that $\partial_{33} v_{\alpha}^{2}$ belongs to $L^{2}\left(I, H^{m-1}(\omega)\right) \cap$ $H^{m-1}\left(I, L^{2}(\omega)\right)$, whence $v_{\alpha}^{2} \in H^{2}\left(I, H^{m-1}(\omega)\right) \cap H^{m+1}\left(I, L^{2}(\omega)\right)$.

As a consequence the trace of $v_{3}^{2}$ on $\Gamma_{0}$ belongs to $H^{1 / 2}\left(I, H^{m-1 / 2}(\partial \omega)\right)$. Thus the traces $c_{t}, c_{s}, c_{n} c f$. (4.4) and (4.5), of the generating functions of $u_{\mathrm{KL}}^{1}$ satisfy

$$
\left.\zeta_{\alpha}^{1}\right|_{\partial \omega} \in H^{m-1 / 2}(\partial \omega) \text { and }\left.\quad \partial_{n} \zeta_{3}^{1}\right|_{\partial \omega} \in H^{m-1 / 2}(\partial \omega) .
$$

Whence by solution of the membrane and bending problems:

$$
\zeta_{\alpha}^{1} \in H^{m}(\omega) \quad \text { and } \quad \zeta_{3}^{1} \in H^{m+1}(\omega)
$$


Returning to the "even Ansatz", we also deduce from the regularity of $v^{2}$ that $e_{\gamma 3}\left(v^{2}\right)$ belongs to $H^{1}\left(I, H^{m-1}(\omega)\right) \cap H^{m}\left(I, L^{2}(\omega)\right)$, whence the right hand side $F_{\alpha}\left(v^{2}\right)$ of problem (2.19) is in $H^{m-3}(\omega)$ and by elliptic regularity $\zeta_{\alpha}^{2}$ is in $H^{m-1}(\omega)$. Going on, we obtain that, by solution of the Neumann problems (2.15b)-(2.15c) and (2.16b)-(2.16c), $y_{3}^{4}$ belongs to $H^{3}\left(I, H^{m-2}(\omega)\right) \cap H^{m+1}\left(I, L^{2}(\omega)\right)$ and $y_{\alpha}^{4}$ to $H^{4}\left(I, H^{m-3}(\omega)\right) \cap H^{m+1}\left(I, L^{2}(\omega)\right)$. Whence $e_{\gamma 3}\left(y^{4}\right)$ belongs to $H^{3}\left(I, H^{m-3}(\omega)\right) \cap$ $H^{m}\left(I, L^{2}(\omega)\right)$ and the right hand side $F_{3}\left(y^{4}\right)$ of problem $(2.22)$ to $H^{m-4}(\omega)$. Therefore $\zeta_{3}^{2}$ belongs to $H^{m}(\omega)$.

Going on, we prove

Lemma 4.2 Under the assumption (4.16), we have for any $0 \leq k \leq m$

$$
\zeta_{\alpha}^{k} \in H^{m+1-k}(\omega) \quad \text { and } \quad \zeta_{3}^{k} \in H^{m+2-k}(\omega)
$$

and for $2 \leq k \leq m$

$$
\left\{\begin{array}{l}
v_{\alpha}^{k} \in H^{k}\left(I, H^{m+1-k}(\omega)\right) \cap H^{m+1}\left(I, L^{2}(\omega)\right) \\
v_{3}^{k} \in H^{k-1}\left(I, H^{m+2-k}(\omega)\right) \cap H^{m+1}\left(I, L^{2}(\omega)\right),
\end{array}\right.
$$

or, equivalently

$$
\begin{cases}\partial_{3}^{\ell} v_{\alpha}^{k} \in H^{m+1-k}(\Omega), & 0 \leq \ell \leq k \\ \partial_{3}^{\ell} v_{3}^{k} \in H^{m+2-k}(\Omega), & 0 \leq \ell \leq k-1 .\end{cases}
$$

Of course, if $f$ is $\mathscr{C}^{\infty}(\bar{\Omega})$, all the terms $u_{\mathrm{KL}}^{k}$ and $v_{k}$ of the polynomial Ansatz are also $\mathscr{C}^{\infty}(\bar{\Omega})$. On the other hand, the regularity of the profiles $w^{k}$ is severely limited by the singularities along the edges of the plate (see Part II).

\section{ERROR ESTIMATES IN ENERGY NORM}

\subsection{The main error estimate.}

For the justification of an asymptotic expansion, two methods may be considered according as to whether one knows only the leading term or one can compute terms of arbitrarily high orders. The first one has given rise to numerous articles on the subject ( $c f$. . [8], [7] and the references that we quoted in $\S 1.3)$ and results are by now classical: In [7], it is shown that the three-dimensional solution $u(\varepsilon)$ of the scaled problem converges for the topology of $H^{1}(\Omega)$ towards the leading term $u^{0}$ of the Ansatz. There, no mention is made about the way it converges, except in $[14,15,17]$ where an estimation of the error is obtained.

The other method which is actually considered here, uses the formal asymptotic expansion (4.6) which is valid up to an arbitrarily high order $N$. It yields an optimal estimation of the error between the scaled displacement $u(\varepsilon)$ and the Ansatz of order $N$. With the notations already used in Theorem 4.1, we state the main result: 
Theorem 5.1 Let $u(\varepsilon)$ be the solution of problem (1.6). There holds $\forall N \geq 0$ :

$$
\left\|u(\varepsilon)-\sum_{k=0}^{N} \varepsilon^{k} u^{k}+\chi(r) \sum_{k=1}^{N} \varepsilon^{k} w^{k}\left(\frac{r}{\varepsilon}, s, x_{3}\right)\right\|_{H^{1}(\Omega)^{3}} \leq C \varepsilon^{N+1 / 2}
$$

where $u^{k}=u_{\mathrm{KL}}^{k}+v^{k}$ and with $u_{\mathrm{KL}}^{k}, v^{k}, w^{k}$ and $\chi$ as defined in Theorem 4.1.

As developed in the next section, the proof of this theorem relies on energy estimates, for which we do not try to reach optimality, and on a very simple argument consisting in pushing the development a few terms further and relying on the following estimates of each term in the asymptotics:

$$
\left\|u^{k}\right\|_{H^{1}(\Omega)} \leq C \quad \text { and } \quad\left\|\chi(r) w^{k}\left(\frac{r}{\varepsilon}, s, x_{3}\right)\right\|_{H^{1}(\Omega)} \leq C \varepsilon^{-1 / 2} .
$$

The corresponding $L^{2}$ - estimates are

$$
\left\|u^{k}\right\|_{L^{2}(\Omega)} \leq C \quad \text { and } \quad\left\|\chi(r) w^{k}\left(\frac{r}{\varepsilon}, s, x_{3}\right)\right\|_{L^{2}(\Omega)} \leq C \varepsilon^{1 / 2} .
$$

So, we can deduce in a straightforward way the $L^{2}$ - estimates (1.14c) and (1.14d) for the displacement and (1.18) for the strain tensor from Theorem 5.1 and from (5.2)-(5.3).

\subsection{Proof of Theorem (5.1)}

Setting:

$$
\bar{U}^{N}(\varepsilon)=u(\varepsilon)-U^{N}(\varepsilon)
$$

where $U^{N}(\varepsilon)$ denotes the asymptotic expansion of order $N$, namely:

$$
U^{N}(\varepsilon)=\sum_{k=0}^{N} \varepsilon^{k} u^{k}-\chi(r) \sum_{k=1}^{N} \varepsilon^{k} w^{k}\left(\frac{r}{\varepsilon}, s, x_{3}\right)
$$

we see that it is sufficient to establish an a priori estimate for the remainder $\bar{U}^{N}(\varepsilon)$ in the norm of the space $H^{1}(\Omega)^{3}$. Thanks to the coercivity of the elasticity operator and to Korn's inequality which holds for the elements of the space $V(\Omega)$, this will follow from an energy method which consists in an estimation of the energy associated with the remainder, namely:

$$
\int_{\Omega} A \kappa(\varepsilon)\left(\bar{U}^{N}(\varepsilon)\right): \kappa(\varepsilon)\left(\bar{U}^{N}(\varepsilon)\right) .
$$

(i) The Energy method. We split $U^{N}(\varepsilon)$ into its two natural parts:

$$
U^{N}(\varepsilon)=V^{N}(\varepsilon)-\chi(r) W^{N}(\varepsilon)
$$

where

$$
V^{N}(\varepsilon)=\sum_{k=0}^{N} \varepsilon^{k} u^{k} \quad \text { and } \quad W^{N}(\varepsilon)=\sum_{k=1}^{N} \varepsilon^{k} w^{k}\left(\frac{r}{\varepsilon}, s, x_{3}\right) .
$$

A close look at the construction of the complete asymptotics, in particular to (4.10), (4.14) and (4.15), allows to prove: 
Lemma 5.2 For any $N \in \mathbb{N}, U^{N}(\varepsilon)$ belongs to the space $V(\Omega)$ of admissible displacements satisfying the Dirichlet condition on the clamped part of the boundary.

Thus, we have

$$
\forall N \in \mathbb{N}, \quad \bar{U}^{N}(\varepsilon) \in V(\Omega),
$$

and $\bar{U}^{N}(\varepsilon)$ is the solution of the problem:

$$
\begin{aligned}
& \bar{U}^{N}(\varepsilon) \in V(\Omega), \\
& \forall v \in V(\Omega),\left\{\begin{aligned}
\int_{\Omega} A \kappa(\varepsilon)\left(\bar{U}^{N}(\varepsilon)\right): & \kappa(\varepsilon)(v)=\int_{\Omega}\left(F^{N}(\varepsilon)-G^{N}(\varepsilon)\right) \cdot v \\
& +\int_{\partial \Omega \cap\left\{x_{3}= \pm 1\right\}}\left(f^{N}(\varepsilon)-g^{N}(\varepsilon)\right) \cdot v
\end{aligned}\right.
\end{aligned}
$$

where we have decomposed the error into the two following contributions: for all $v \in V(\Omega)$,

$$
\int_{\Omega} A \kappa(\varepsilon)\left(u(\varepsilon)-V^{N}(\varepsilon)\right): \kappa(\varepsilon)(v)=\int_{\Omega} F^{N}(\varepsilon) \cdot v+\int_{\partial \Omega \cap\left\{x_{3}= \pm 1\right\}} f^{N}(\varepsilon) \cdot v,
$$

and

$$
\int_{\Omega} A \kappa(\varepsilon)\left(\chi(r) W^{N}(\varepsilon)\right): \kappa(\varepsilon)(v)=\int_{\Omega} G^{N}(\varepsilon) \cdot v+\int_{\partial \Omega \cap\left\{x_{3}= \pm 1\right\}} g^{N}(\varepsilon) \cdot v .
$$

In particular, for the choice $v=\bar{U}^{N}(\varepsilon)$ of the test function, we obtain the energy of the elasticity problem as the left-hand side of the variational equation $(5.9 \mathrm{~b})$. Korn's inequality and the coercivity of the operator of elasticity will allow the method of energy to provide the following rough estimates.

\section{Lemma 5.3}

$$
\left\|\bar{U}^{N}(\varepsilon)\right\|_{H^{1}(\Omega)^{3}} \leq C \varepsilon^{N-3} .
$$

Proof. We have to estimate the right hand sides of (5.10) and (5.11).

Considering the equations (4.8) and (4.9) satisfied by $u^{k}$, and noting that the power of $\varepsilon$ multiplying (4.8) is $\varepsilon^{k-4}$ and the one multiplying (4.9) is $\varepsilon^{k-2}$, we obtain that:

$$
F^{N}(\varepsilon)=\mathcal{O}\left(\varepsilon^{N-3}\right) \text { and } \quad f^{N}(\varepsilon)=\mathcal{O}\left(\varepsilon^{N-3}\right) .
$$

Concerning (5.11), we first note

$$
\begin{aligned}
& \mid \int_{\Omega} A \kappa(\varepsilon)\left(\chi W^{N}(\varepsilon)\right): \kappa(\varepsilon)(v)-\int_{\Omega} A \kappa(\varepsilon)\left(W^{N}(\varepsilon)\right): \kappa(\varepsilon)(\chi v) \mid \leq \\
& \leq C \varepsilon^{-1}\left(\left\|\chi^{\prime} W^{N}\right\|_{L^{2}}\|\kappa(\varepsilon)(v)\|_{L^{2}}+\|v\|_{L^{2}}\left\|\chi^{\prime} \kappa(\varepsilon)\left(W^{N}\right)\right\|_{L^{2}}\right) .
\end{aligned}
$$

Taking advantage of the fact that $w^{k}$, or equivalently $\varphi^{k}$, is an element of the space $\boldsymbol{K}_{\eta_{0}}^{1}\left(\Sigma^{+}\right)$, (see Definition 3.3), we obtain:

$$
\exists c>0, \gamma>0: \quad\left\|\chi^{\prime} W^{N}\right\|_{L^{2}}+\left\|\chi^{\prime} \kappa(\varepsilon)\left(W^{N}\right)\right\|_{L^{2}} \leq c e^{-\gamma / \varepsilon} .
$$


Thus, there exist $c^{\prime}>0$ and $\gamma^{\prime}>0$, such that:

$$
\left|\int_{\Omega} A \kappa(\varepsilon)\left(\chi W^{N}\right): \kappa(\varepsilon)(v)-\int_{\Omega} A \kappa(\varepsilon)\left(W^{N}\right): \kappa(\varepsilon)(\chi v)\right| \leq c^{\prime} e^{-\gamma^{\prime} / \varepsilon}\|v\|_{H^{1}} .
$$

Setting

$$
\tilde{v}\left(t, s, x_{3}\right)=\varepsilon^{-1}\left(\varepsilon \chi v_{*}, \chi v_{3}\right)\left(r, s, x_{3}\right) \quad \text { and } \quad \Phi^{N}\left(t, s, x_{3}\right)=\left(\varepsilon W_{*}^{N}, W_{3}^{N}\right)\left(r, s, x_{3}\right)
$$

and relying on $(3.6 \mathrm{~b}),(3.7 \mathrm{~b})$ and $(4.15)$, we obtain

$$
\left|\int_{\widetilde{\Sigma}} \widetilde{A}(\varepsilon t, s) e\left(\partial_{t}, 0, \partial_{3}\right)\left(\Phi^{N}\right): e\left(\partial_{t}, 0, \partial_{3}\right)(\tilde{v})+\sum_{k=1}^{4} \varepsilon^{k} \widetilde{\mathcal{A}}^{k}\left(\Phi^{N}, \tilde{v}\right)\right|=\mathcal{O}\left(\varepsilon^{N+1}\right)\|\tilde{v}\|_{H^{1}(\widetilde{\Sigma})},
$$

whence, with (3.4b):

$$
\left|\int_{\Omega} A \kappa(\varepsilon)\left(W^{N}(\varepsilon)\right): \kappa(\varepsilon)(\chi v)\right|=\mathcal{O}\left(\varepsilon^{N-1 / 2}\right)\|v\|_{H^{1}(\Omega)} .
$$

Putting (5.14) and (5.15) together, we have:

$$
\left|\int_{\Omega} A \kappa(\varepsilon)\left(\chi W^{N}(\varepsilon)\right): \kappa(\varepsilon)(v)\right|=\mathcal{O}\left(\varepsilon^{N-1 / 2}\right)\|v\|_{H^{1}(\Omega)} .
$$

Now, from (5.9) with (5.10), (5.11), (5.13) and (5.16), we may deduce an upper bound for the elastic energy associated with the remainder $\bar{U}^{N}(\varepsilon)$ :

$$
\int_{\Omega} A \kappa(\varepsilon)\left(\bar{U}^{N}(\varepsilon)\right): \kappa(\varepsilon)\left(\bar{U}^{N}(\varepsilon)\right) \leq C \varepsilon^{N-3}\left\|\bar{U}^{N}(\varepsilon)\right\|_{H^{1}(\Omega)}
$$

and we get from Korn's inequality, as $\bar{U}^{N}(\varepsilon)$ is an element of the space $V(\Omega)$ :

$$
\int_{\Omega} A \kappa(\varepsilon)\left(\bar{U}^{N}(\varepsilon)\right): \kappa(\varepsilon)\left(\bar{U}^{N}(\varepsilon)\right) \leq C \varepsilon^{N-3}\left\|\kappa(\varepsilon)\left(\bar{U}^{N}(\varepsilon)\right)\right\|_{L^{2}(\Omega)} .
$$

We use Korn's inequality again and the positivity of the matrix $A$ to conclude.

(ii) Improvement of the estimate. Lemma 5.3 yields the following estimate for $\bar{U}^{N+4}(\varepsilon)$ :

whence:

$$
\left\|\bar{U}^{N+4}(\varepsilon)\right\|_{H^{1}(\Omega)} \leq C \varepsilon^{N+1}
$$

$$
\begin{aligned}
\left\|u(\varepsilon)-\sum_{k=0}^{N} \varepsilon^{k} u^{k}+\chi(r) \sum_{k=1}^{N} \varepsilon^{k} w^{k}\left(\frac{r}{\varepsilon}, s, x_{3}\right)\right\|_{H^{1}(\Omega)^{3}} \leq \\
\leq C \varepsilon^{N+1}+\sum_{k=N+1}^{N+4} \varepsilon^{k}\left(\left\|u^{k}\right\|_{H^{1}(\Omega)^{3}}+\left\|\chi(r) w^{k}\left(\frac{r}{\varepsilon}, s, x_{3}\right)\right\|_{H^{1}(\Omega)^{3}}\right) .
\end{aligned}
$$

Relying on the estimates (5.2) of each term $u^{k}$ and $w^{k}$ for $N+1 \leq k \leq N+4$, the estimate (5.1) directly follows from (5.20).

\section{REFERENCES}

[1] G. Allaire. Homogénéisation et convergence à deux échelles . application à un problème de convection diffusion. C. R. Acad. Sc. Paris, Sér. I 312 (1991) 581-586. 
[2] G. Allaire. Homogenization and two-scale convergence. SIAM J. Math. Anal. 23 (6) (1992) 1482-1518.

[3] D. N. Arnold, R. S. Falk. The boundary layer for the Reissner-Mindlin plate model. SIAM J. Math. Anal. 21 (2) (1990) 281-312.

[4] I. Babuška, J. M. D'Harcourt, C. Schwab. Optimal shear correction factors in hierarchic plate modelling. Math. Modelling and Sc. Computing 1 (1991) 1-30.

[5] D. Caillerie. Models of thin or thick plates and membranes derived from linear elasticity. In P. G. Ciarlet, E. Sanchez-Palencia, editors, Applications of Multiple Scaling in Mechanics, R. M. A. Masson, Paris 1987.

[6] P. G. Ciarlet. Mathematical Elasticity. Vol. I, Three-Dimensional Elasticity. North-Holland, Amsterdam 1988.

[7] P. G. Ciarlet. Plates and Junctions in Elastic Multi-Structures: An Asymptotic Analysis. R.M.A. Vol. 14. Masson and Springer-Verlag, Paris and Heidelberg 1990.

[8] P. G. Ciarlet, P. Destuynder. A justification of the two-dimensional plate model. J. Mécanique 18 (1979) 315-344.

[9] P. G. Ciarlet, S. Kesavan. Two-dimensional approximation of three-dimensional eigenvalue problems in plate theory. Comp. Methods Appl. Mech. Engrg. 26 (1981) 149-172.

[10] P. G. Ciarlet, H. Le Dret, R. Nzengwa. Junctions between threedimensional and two-dimensional linearly elastic structures. J. Math. Pures Appl. 68 (1989) 261-295.

[11] P. G. Ciarlet, V. Lods. Ellipticité des équations membranaires d'une coque uniformément elliptique. C. R. Acad. Sci. Paris, Sér. I 318 (1994) 195-200.

[12] P. G. Ciarlet, V. Lods. Analyse asymptotique des coques linéairement élastiques. I. Coques "membranaires". C. R. Acad. Sci. Paris, Sér. I 318 (1994) $863-868$.

[13] P. G. Ciarlet, V. Lods, B. Miara. Analyse asymptotique des coques linéairement élastiques. II. Coques en "en flexion". C. R. Acad. Sci. Paris, Sér. I 319 (1994) 95-100.

[14] P. Destuynder. Sur une justification des modèles de plaques et de coques par les méthodes asymptotiques. Thèse d'Etat, Université Pierre et Marie Curie, Paris 1980.

[15] P. Destuynder. Comparaison entre les modèles tridimensionnels et bidimensionnels de plaques en élasticité. R.A.I.R.O. Analyse Numérique 15 (1981) $331-369$.

[16] P. Destuynder. Une théorie asymptotique des plaques minces en élasticité linéaire. Masson, Paris 1986. 
[17] P. Destuynder, I. Gruais. Modèle de Kirchhoff-Love : estimation de l'erreur dans le cas encastré $\left(\gamma=\gamma_{0}\right)$ pour le problème de Dirichlet. In P. CiARlet, L. Trabucho, J. V. No, editors, Proceedings of the International Conference on Asymptotic Methods, Lisbonne, Oct. 1993. Birkhäuser, Basel 1995.

[18] K. O. Friedrichs, R. F. Dressler. A boundary-layer theory for elastic plates. Comm. Pure Appl. Math. 14 (1961) 1-33.

[19] A. L. Gol'Denveizer. Derivation of an approximate theory of bending of a plate by the method of asymptotic integration of the equations of the theory of elasticity. Prikl. Math. Mekh. 26 (1962) 668-686. English translation J. Appl. Math. Mech. (1964) 1000-1025.

[20] R. D. Gregory, F. Y. Wan. Decaying states of plane strain in a semi-infinite strip and boundary conditions for plate theory. J. Elasticity 14 (1984) 27-64.

[21] I. Gruais. Modélisation de la jonction entre une plaque et une poutre en élasticité linéarisée. Modél. Math. Anal. Numér. 27 (1993) 77-109.

[22] O. GuÈs. Couches limites pour des problèmes mixtes hyperboliques. Séminaire E. D. P. 17, Centre de Math., Ecole Polytechnique, Palaiseau 1994.

[23] O. Guès. Perturbations singulières de problèmes mixtes hyperboliques multidimensionnels. Preprint, IRMAR, Université de Rennes I, Rennes 1994.

[24] A. M. IL'IN. Matching of asymptotic expansions of solutions of boundary value problems, volume 102 of Translations of Mathematical Monographs. American Mathematical Society, Providence, R. I. 1992.

[25] H. LE Dret. Folded plates revisited. Comput. Mech. 5 (1989) 345-365.

[26] H. Le Dret. Modeling of the junction between two rods. J. Math. Pures Appl. 68 (1989) 365-397.

[27] H. Le Dret. Modeling of a folded plate. Comput.Mech. 5 (1990) 401-416.

[28] J.-L. Lions. Perturbations Singulières dans les Problèmes aux Limites et en Contrôle Optimal. Lectures Notes in Mathematics, Vol. 323. Springer-Verlag, Heidelberg 1973.

[29] B. Miara, E. Sanchez-Palencia. Asymptotic analysis of linearly elastic shells. To appear (1994).

[30] P. M. Naghdi. The theory of shells and plates. In S. Flügge, C. TruesDELL, editors, Handbuch der Physik, volume VI a/2, pages 425-640. SpringerVerlag, Berlin 1972.

[31] S. A. NAzArov. The spatial structure of the stress field in the neighbourhood of the corner point of a thin plate. J. Appl. Maths Mechs 55 (4) (1991) 523-530.

[32] S. A. Nazarov, I. S. Zorin. Egde effect in the bending of a thin threedimensional plate. P. M. M. USSR 53 (4) (1989) 500-507.

[33] O. A. Oleinik, A. S. Shamaev, G. A. Yosifian. Mathematical Problems in Elasticity and Homogenization. Studies in mathematics and its applications. North-Holland, Amsterdam 1992. 
[34] J. C. Paumier. Existence and convergence of the expansion in the asymptotic theory of elastic thin plates. Math. Modelling Numer. Anal. 25 (3) (1990) 371-391.

[35] E. Sanchez-Palencia. Statique et dynamique des coques minces. I. Cas de flexion pure non inhibée. C. R. Acad. Sci. Paris, Sér. I 309 (1989) 411-417.

[36] E. Sanchez-Palencia. Statique et dynamique des coques minces. II. Cas de flexion pure inhibée. Approximation membranaire. C. R. Acad. Sci. Paris, Sér. I 309 (1989) 531-537.

[37] E. Sanchez-Palencia. Passage à la limite de l'élasticité tridimensionnelle à la théorie asymptotique des coques minces. C. R. Acad. Sci. Paris, Sér. II 311 (1990) 909-916.

[38] C. Schwab. A-posteriori modeling error estimation for hierarchic plate models. Preprint, University of Maryland, Baltimore, Maryland 1994.

[39] C. Schwab. Boundary layer resolution in hierarchical models of laminated composites. Math. Model. Numer. Anal. 28 (5) (1994) 517-537. 